\title{
Homogenization of a Ginzburg-Landau problem in a perforated domain with mixed boundary conditions
}

\author{
Luisa Faella ${ }^{1 \dagger}$ and Carmen Perugia ${ }^{2 * \dagger}$
}

\author{
"Correspondence: \\ cperugia@unisannio.it \\ 2Dipartimento di Scienze e \\ Tecnologie, Universitá del Sannio \\ Via Dei Mulini 59/A Palazzo \\ Inarcassa, Benevento, 82100, Italia \\ † Equal contributors \\ Full list of author information is \\ available at the end of the article
}

\begin{abstract}
In this paper we study the asymptotic behavior of a Ginzburg-Landau problem in a $\varepsilon$-periodically perforated domain of $\mathbf{R}^{n}$ with mixed Dirichlet-Neumann conditions. The holes can verify two different situations. In the first one they have size $\varepsilon$ and a homogeneous Dirichlet condition is posed on a flat portion of each hole, whose size is an order smaller than $\varepsilon$, the Neumann condition being posed on the remaining part. In the second situation, we consider two kinds of $\varepsilon$-periodic holes, one of size of order smaller than $\varepsilon$, where a homogeneous Dirichlet condition is prescribed and the other one of size $\varepsilon$, on which a non-homogeneous Neumann condition is given. Moreover, in this case as $\varepsilon$ goes to zero, the two families of holes approach each other. In both situations a homogeneous Dirichlet condition is also prescribed on the whole exterior boundary of the domain.
\end{abstract}

MSC: Primary 35J20; 35J25; 35B25; 35J55; 35B40

Keywords: homogenization; harmonic capacity; extension operator; Ginzburg-Landau equations

\section{Introduction}

Let $\Omega$ be a bounded set in $\mathbf{R}^{n}$, with Lipschitz boundary $\partial \Omega$ and $Y$ be $\left[-\frac{1}{2}, \frac{1}{2}\right]^{n}$. We consider two kinds of holes removed from $\Omega$, both periodically distributed. The first kind is of size $\varepsilon, \varepsilon$ being a positive parameter. It is obtained by rescaling a reference hole $Q$ (a cube or a smoothed one) contained in $Y$ and in the half plane $\left\{x_{1} \geq 0\right\}$, a piece of which is on the hyperplane $\left\{x_{1}=0\right\}$. The latter kind is of size $\varepsilon^{\frac{n}{n-2}}$ if $n \geq 3\left(\exp \left(-\varepsilon^{-2}\right)\right.$ if $\left.n=2\right)$ and it is obtained by rescaling a reference hole $K$ strictly contained in $Y$ and in the half plane $x_{1} \leq 0$ (and containing a segment on the line $x_{1}=0$ if $n=2$ ). Moreover, every element of the second family moves perpendicularly with respect to the $x_{n}$-axis towards an element of the first family with an approaching speed of order $\tau \varepsilon^{\sigma}$ if $n \geq 3\left(\exp \left(-1 / \varepsilon^{\sigma}\right) \tau\right.$ if $n=2)$ where $\tau=\left(\tau_{1}, 0, \ldots, 0\right) \in \mathbf{R}^{n}, \tau_{1} \leq 0$. Then we assign a non-homogeneous Neumann condition on the first family so that there is no total flow in $\Omega$ through these holes and a homogeneous Dirichlet condition on the latter family. We observe explicitly that if $K$ is contained in the set $\left\{x \in \mathbf{R}^{n}: x_{1}=0\right\}$ and we take $\tau=0$ we obtain the case when the zone where homogeneous Dirichlet condition is imposed, lies exactly on the boundary of the holes of the first family. Let $\Omega_{\varepsilon}^{\tau, \sigma}$ be the set obtained by removing from $\Omega$ the two families of holes previously described. In this paper we study the homogenization process of the

(c) 2014 Faella and Perugia; licensee Springer. This is an Open Access article distributed under the terms of the Creative Commons Attribution License (http://creativecommons.org/licenses/by/4.0), which permits unrestricted use, distribution, and reproduction in any medium, provided the original work is properly credited. 
following vectorial nonlinear problem with mixed boundary conditions:

$$
\begin{cases}-\Delta u_{\varepsilon}^{\tau, \sigma}-u_{\varepsilon}^{\tau, \sigma}+\left|u_{\varepsilon}^{\tau, \sigma}\right|^{2} u_{\varepsilon}^{\tau, \sigma}=f, & \text { in } \Omega_{\varepsilon}^{\tau, \sigma}, \\ u_{\varepsilon}^{\tau, \sigma}=0, & \text { on } \partial \Omega, \\ \text { described conditions on } \partial \Omega_{\varepsilon}^{\tau, \sigma} \backslash \partial \Omega . & \end{cases}
$$

Let us observe that the equation in (1.1) is known as the Ginzburg-Landau equation.

By using the energy method (see [1]), for $n=2$ and $n=3$ we prove the weakly convergence in $H_{0}^{1}(\Omega)$ of a suitable extension of the sequence of solutions $u_{\varepsilon}^{\tau, \sigma}$ of $(1.1)$ to a function $u^{\tau, \sigma}$, unique solution of the following limit problem:

$$
\begin{cases}-\operatorname{div} \mathcal{A}\left(\nabla u^{\tau, \sigma}\right)+\frac{1}{2} \mu^{\tau, \sigma} u^{\tau, \sigma}-u^{\tau, \sigma}+\left|u^{\tau, \sigma}\right|^{2} u^{\tau, \sigma}=\theta f, & \text { in } \Omega, \\ u^{\tau, \sigma}=0, & \text { on } \partial \Omega\end{cases}
$$

where $\mathcal{A}$ is the standard homogenized matrix which appears in [2] and $\theta$ is, roughly speaking, the volume of the 'material' in $\Omega$. Furthermore, the definition of $\mu^{\tau, \sigma}$ depends both on $\sigma$ or on the dimension $n$. More precisely

if $n=2, \mu^{\tau, \sigma}$ is equal to $4 \pi$ if $\sigma<2$ and $2 \pi$ if $\sigma \geq 2$;

if $n=3, \mu^{\tau, \sigma}$ is equal to

(a) the double of the capacity in $\mathbf{R}^{3}$ of the Dirichlet reference hole if $1 \leq \sigma<3$;

(b) the capacity in $\mathbf{R}^{3}$ of a set obtained by perpendicularly translating the Dirichlet reference hole to a distance $\tau=\left(\tau_{1}, 0,0\right) \in \mathbf{R}^{3}, \tau_{1} \leq 0$ from the hyperplane $\left\{x_{1}=0\right\}$ and after doubling it by reflection with respect to the same hyperplane if $\sigma=3$;

(c) the capacity in $\mathbf{R}^{3}$ of a set obtained by doubling the Dirichlet reference hole by reflection with respect to the hyperplane $\left\{x_{1}=0\right\}$ if $\sigma>3$.

We observe explicitly that no additional term of flow appears in (1.2). This is a consequence of the fact that there is no total flow. Eventually we observe that in the case (a) the term $\frac{1}{2} \mu^{\tau, \sigma}$ is exactly the 'strange term' of [3] and in the case (b) it is exactly the half of this quantity.

The paper is organized as follows. In Section 2, we describe the domain with appropriate spaces required and we give the main result. In Section 3, we recall some preliminary lemmas and prove an important result involving the capacity in $\mathbf{R}^{n}$ of the Dirichlet reference hole. Finally, Section 4 is devoted to the proof of the main theorem, by establishing some a priori norm estimates for the sequence of solutions and convergence results.

Many authors (see for example [2-10]) studied the asymptotic behavior, as $\varepsilon$ tends to zero, of solutions of scalar boundary value problems defined in a domain $\Omega_{\varepsilon}$ obtained by removing from $\Omega$ closed smoothed cubes well contained in $\Omega$ (the holes) of diameter $r(\varepsilon) \leq \varepsilon$ periodically distributed with period $\varepsilon$ in $\mathbf{R}^{n}$. In particular in [10] is studied, perhaps for the first time, a problem in which both Neumann and Dirichlet conditions are present on the boundary of the holes. The Dirichlet condition is given on a flat portion of diameter $\varepsilon^{\frac{n}{n-2}}$ if $n \geq 3\left(\exp \left(-\varepsilon^{-2}\right)\right.$ if $\left.n=2\right)$, the Neumann condition is non-homogeneous so the reference hole has to be rescaled with $r(\varepsilon)=\varepsilon^{\frac{n}{n-1}}$. In [5] a problem where both Neumann and Dirichlet conditions are present on the boundary of the holes is also studied, where, the Neumann condition being homogeneous, the reference hole is rescaled with $r(\varepsilon)=\varepsilon$. On the other side, an extensive study of Ginzburg-Landau equation in a bounded 
domain $\Omega$ of $\mathbf{R}^{2}$ is performed by several authors starting from the pioneering papers of Bethuel, Brezis and Hélein (see for example [11-18]). The limit behavior of the GinzburgLandau equation in a perforated domain in $\mathbf{R}^{3}$ with holes along a plane is studied in [19] while in [20] is studied the homogenization of the Ginzburg-Landau equation in a domain of $\mathbf{R}^{2}$ with oscillating boundary.

\section{Statement of the problem and main result}

Let $\Omega$ be an open bounded subset of $\mathbf{R}^{n}$ with Lipschitz boundary. Let $Y=[-1 / 2,1 / 2]^{n} \subset \mathbf{R}^{n}$ and $l>0$ such that the cube $R=[0,2 l] \times[-l, l]^{n-1} \subset \stackrel{\circ}{Y}$. For $n=2$, or $n=3$ we take $Q=R$. For $n>3$ we consider a domain $Q$ which has $C^{\infty}$ boundary such that

$$
\frac{3}{4} R \subseteq Q \subseteq R
$$

Let us observe that $\partial Q \supseteq \partial\left(\frac{3}{4} R\right) \cap \partial R$. Let us pose $Y^{c}=Y \backslash Q$. Let $K$ be a compact subset of $\mathbf{R}^{n}$, contained in the half plane $x_{1} \leq 0$ and in $Y$; moreover, if $n=2$ let $K \cap\left\{x \in \mathbf{R}^{n}: x_{1}=0\right\}$ contain a segment.

Let $f=\left(f_{1}, f_{2}\right) \in\left(L^{2}(\Omega)\right)^{2}$ and let $g=\left(g_{1}, g_{2}\right) \in\left(L^{2}(\partial Q)\right)^{2}$ be a null average function.

Let $\varepsilon>0$ and $Y_{\varepsilon}=Y_{\varepsilon}(\Omega)=\bigcup\left\{\varepsilon(Y+\mathbf{k}): \mathbf{k} \in \mathbf{Z}^{n}\right.$ and $\left.\varepsilon(Y+\mathbf{k}) \subset \Omega\right\}$. Let $Q_{\varepsilon}=\varepsilon Q$ and

$$
S_{\varepsilon}^{\tau, \sigma}= \begin{cases}\varepsilon^{\frac{n}{n-2}} K+\varepsilon^{\sigma} \tau, & n \geq 3 \\ \exp \left(-1 / \varepsilon^{2}\right) K+\exp \left(-1 / \varepsilon^{\sigma}\right) \tau, & n=2,\end{cases}
$$

where $\tau=\left(\tau_{1}, 0, \ldots, 0\right) \in \mathbf{R}^{n}, \tau_{1} \leq 0$, and $\sigma \geq 1$; let us observe that there exists $\varepsilon_{\tau}>0$ such that if $0<\varepsilon<\varepsilon_{\tau}$ then $S_{\varepsilon}^{\tau, \sigma}$ is well contained in $\varepsilon Y$ (see Figure 1 and Figure 2).

Let us define $T_{\varepsilon}=T_{\varepsilon}(\Omega)=\left(\bigcup\left\{Q_{\varepsilon}+\varepsilon \mathbf{k}: \mathbf{k} \in \mathbf{Z}^{n}\right\}\right) \cap Y_{\varepsilon}$ and $\Omega_{\varepsilon}=\Omega \backslash T_{\varepsilon}$.

Let $g_{\varepsilon}$ be the function defined on $\partial T_{\varepsilon}$ by $g_{\varepsilon}(x)=g\left(\frac{x}{\varepsilon}\right)$ if $x \in \partial\left(Q_{\varepsilon}+\varepsilon \mathbf{k}\right)$ and $k \in \mathbf{Z}^{n}$.

Let $K^{*}=\left\{x \in \mathbf{R}^{n}:\left(-x_{1}, x_{2}, \ldots, x_{n}\right) \in K\right\}$.

Let $D_{\varepsilon}^{\tau, \sigma}=D_{\varepsilon}^{\tau, \sigma}(\Omega)=\left(\bigcup\left\{S_{\varepsilon}^{\tau, \sigma}+\varepsilon \mathbf{k}: \mathbf{k} \in \mathbf{Z}^{n}\right\}\right) \cap Y_{\varepsilon}$ if $0<\varepsilon<\varepsilon_{\tau}, D_{\varepsilon}^{\tau, \sigma}=\emptyset$ otherwise.

Let $\Omega_{\varepsilon}^{\tau, \sigma}=\Omega \backslash\left(T_{\varepsilon} \cup D_{\varepsilon}^{\tau, \sigma}\right)$. Let $\Gamma_{\varepsilon}^{D, \tau, \sigma}=\partial D_{\varepsilon}^{\tau, \sigma}, \Gamma_{\varepsilon}^{N, \tau, \sigma}=\partial T_{\varepsilon} \backslash D_{\varepsilon}^{\tau, \sigma}$ (see Figure 3 and Figure 4).

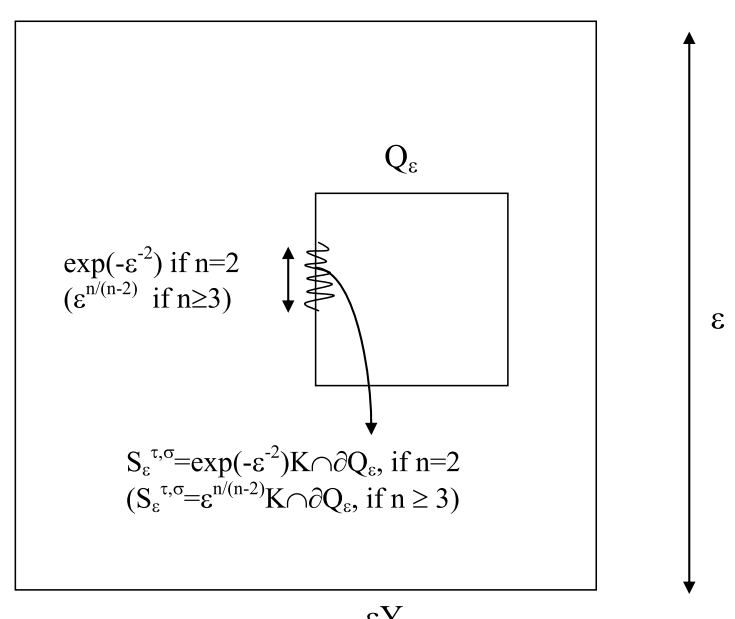

Figure 1 The cell $\varepsilon Y$ : case $\tau=0$. 


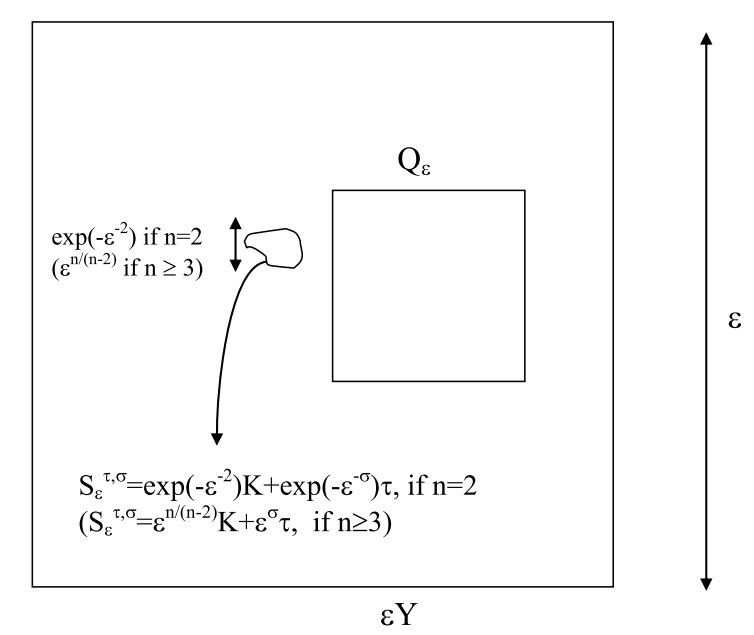

Figure 2 The cell $\varepsilon Y$ : case $\tau \neq 0$.

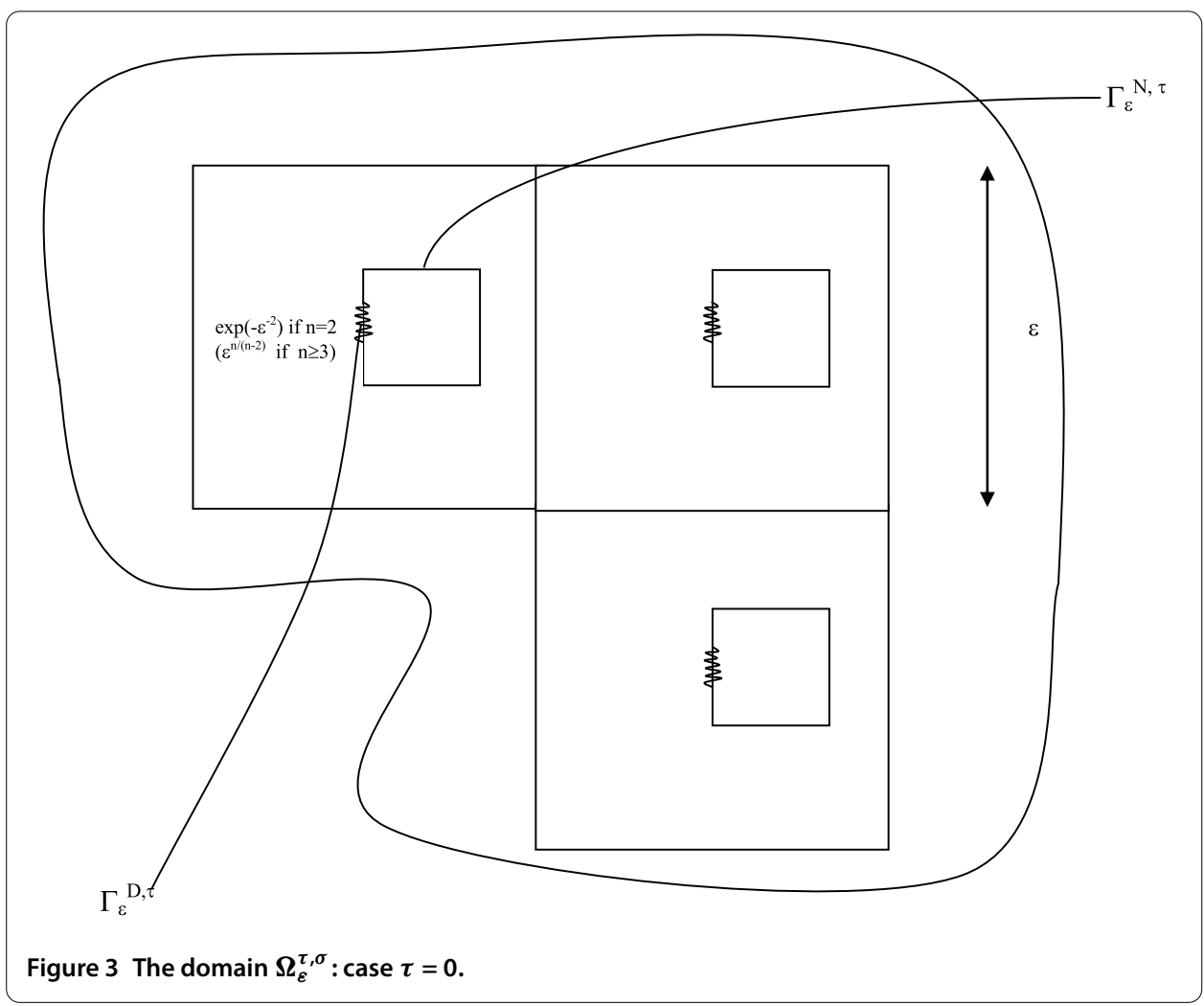

Definition 2.1 Let $K$ be a compact subset of $\mathbf{R}^{n}$ and $\Omega$ an open set such that $K \subset \Omega$. We define the (harmonic) capacity of $K$ with respect to $\Omega$, and we will denote by $\operatorname{cap}(K, \Omega)$ the following quantity:

$$
\operatorname{cap}(K, \Omega)=\inf \left\{\int_{\Omega}|\nabla \varphi|^{2} d x: \varphi \in C_{c}^{1}(\Omega), 0 \leq \varphi \leq 1 \text { and } \varphi \equiv 1 \text { on } K\right\} .
$$

We will, moreover, denote by $\operatorname{cap}(K)$ the quantity $\operatorname{cap}\left(K, \mathbf{R}^{n}\right)$. 


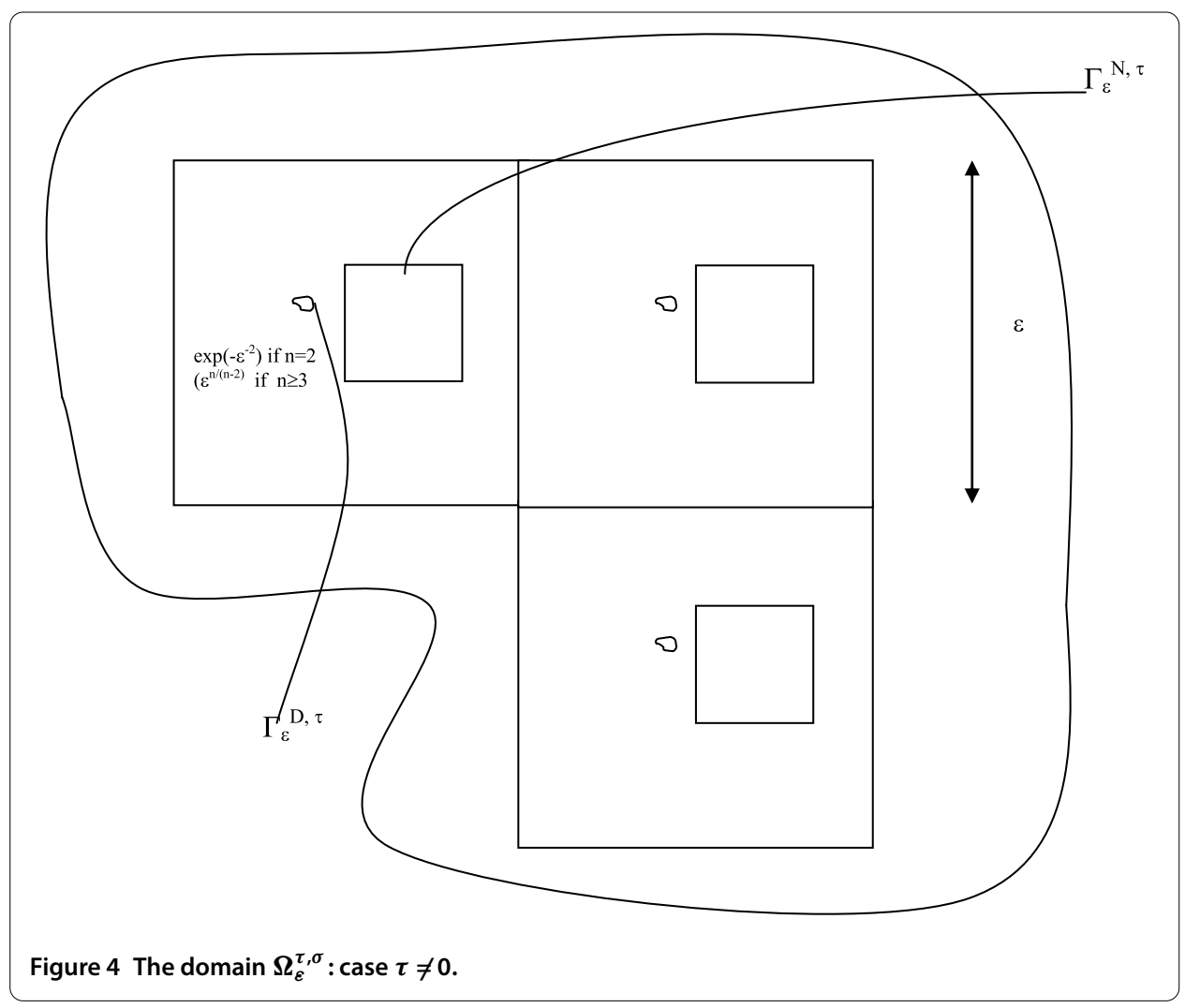

Let us consider, for every $\varepsilon>0$, the following problem:

$$
\begin{cases}-\Delta u_{\varepsilon}^{\tau, \sigma}-u_{\varepsilon}^{\tau, \sigma}+\left|u_{\varepsilon}^{\tau, \sigma}\right|^{2} u_{\varepsilon}^{\tau, \sigma}=f, & \text { in } \Omega_{\varepsilon}^{\tau, \sigma}, \\ u_{\varepsilon}^{\tau, \sigma}=0, & \text { on } \partial \Omega \cup \Gamma_{\varepsilon}^{D, \tau, \sigma}, \\ \frac{\partial u_{\varepsilon}^{\tau, \sigma}}{\partial \mathbf{n}}=g_{\varepsilon}, & \text { on } \Gamma_{\varepsilon}^{N, \tau, \sigma},\end{cases}
$$

whose variational formulation is

$$
\left\{\begin{array}{l}
u_{\varepsilon}^{\tau, \sigma} \in\left(V_{\varepsilon}^{\tau, \sigma}\right)^{2}, \\
\int_{\Omega_{\varepsilon}^{\tau, \sigma}\left\langle\nabla u_{\varepsilon}^{\tau, \sigma}, \nabla \varphi\right\rangle+\int_{\Omega_{\varepsilon}^{\tau, \sigma}}\left|u_{\varepsilon}^{\tau, \sigma}\right|^{2} u_{\varepsilon}^{\tau, \sigma} \varphi-\int_{\Omega_{\varepsilon}^{\tau, \sigma}} u_{\varepsilon}^{\tau, \sigma} \varphi} \quad \int_{\Omega_{\varepsilon}^{\tau, \sigma} f \varphi+\int_{\Gamma_{\varepsilon}^{N, \tau, \sigma}} g_{\varepsilon} \varphi, \quad \forall \varphi \in\left(V_{\varepsilon}^{\tau, \sigma}\right)^{2},}
\end{array}\right.
$$

where $V_{\varepsilon}^{\tau, \sigma}$ denote the closure of $C_{0}^{1}\left(\Omega \backslash \Gamma_{\varepsilon}^{D, \tau, \sigma}\right)$ in $H^{1}\left(\Omega_{\varepsilon}^{\tau, \sigma}\right)$.

For any $\lambda \in \mathbf{R}^{n}$, let $w_{\lambda} \in H^{1}\left(Y^{c}\right)$ be the solution of the following problem:

$$
\begin{cases}-\Delta w_{\lambda}=0, & \text { in } Y^{c} \\ w_{\lambda}(y)-\lambda y, & Y \text {-periodic } \\ \frac{\partial w_{\lambda}}{\partial \mathbf{n}}=0, & \text { on } \partial Q\end{cases}
$$

Since $w_{\lambda}$ is linear in $\lambda$ and the extension operator to zero is linear, we can consider the matrix $\mathcal{A}$ given by

$$
\mathcal{A} \lambda=m_{Y}\left(\widetilde{\nabla w_{\lambda}}\right)=\frac{1}{|Y|} \int_{Y^{c}} \nabla w_{\lambda} d y, \quad \forall \lambda \in \mathbf{R}^{n}
$$


where $\widetilde{\nabla w_{\lambda}}$ denotes the extension to zero of $\nabla w_{\lambda}$ on the whole $Y$. In what follows, with $m_{S}(u)$ we will denote the average of the function $u$ over the subset $S \subset \mathbf{R}^{n}$.

We give the following result.

Theorem 2.1 Let $\varepsilon$ be a parameter taking values in a sequence going to zero and let $\left\{u_{\varepsilon}^{\tau, \sigma}\right\}_{\varepsilon}$ be the sequence of solutions of problem (2.2). Then there exists a bounded sequence $\left\{v_{\varepsilon}^{\tau, \sigma}\right\}_{\varepsilon}$ in $\left(H_{0}^{1}(\Omega)\right)^{2}$ extending $\left\{u_{\varepsilon}^{\tau, \sigma}\right\}_{\varepsilon}$ and weakly converging in $\left(H_{0}^{1}(\Omega)\right)^{2}$, for $n=2$ and $n=3$, to the solution $u^{\tau, \sigma}$ of the following homogenized problem:

$$
\begin{cases}-\operatorname{div}\left(\mathcal{A} \nabla u^{\tau, \sigma}\right)+\frac{1}{2} \mu^{\tau, \sigma} u^{\tau, \sigma}-u^{\tau, \sigma}+\left|u^{\tau, \sigma}\right|^{2} u^{\tau, \sigma}=\theta f & \text { in } \Omega, \\ u^{\tau, \sigma}=0 & \text { on } \partial \Omega\end{cases}
$$

or, in the variational formulation,

$$
\left\{\begin{array}{l}
u^{\tau, \sigma} \in\left(H_{0}^{1}(\Omega)\right)^{2} \\
\int_{\Omega}\left\langle\mathcal{A} \nabla u^{\tau, \sigma}, \nabla \varphi\right\rangle+\frac{1}{2} \mu^{\tau, \sigma} \int_{\Omega} u^{\tau, \sigma} \varphi-\int_{\Omega} u^{\tau, \sigma} \varphi+\int_{\Omega}\left|u^{\tau, \sigma}\right|^{2} u^{\tau, \sigma} \varphi \\
\quad=\int_{\Omega} \theta f \varphi, \quad \forall \varphi \in(\mathcal{D}(\Omega))^{2},
\end{array}\right.
$$

where $\mathcal{A}$ is the constant matrix defined in (2.5), and

$$
\mu^{\tau, \sigma}= \begin{cases}2 \operatorname{cap}(K), & \text { if } 1 \leq \sigma<3 \text { for every } \tau \\ \operatorname{cap}\left((K+\tau) \cup\left(K^{*}-\tau\right)\right), & \text { if } \sigma=3 \\ \operatorname{cap}\left(K \cup K^{*}\right), & \text { if } \sigma>3 \text { for every } \tau\end{cases}
$$

if $n=3$, and

$$
\mu^{\tau, \sigma}= \begin{cases}4 \pi, & \text { if } \sigma<2, \\ 2 \pi, & \text { if } \sigma \geq 2,\end{cases}
$$

if $n=2$.

Moreover, any sequence of functions bounded in $\left(H_{0}^{1}(\Omega)\right)^{2}$ and extending $\left\{u_{\varepsilon}^{\tau, \sigma}\right\}_{\varepsilon}$ converges to $u^{\tau, \sigma}$.

\section{Preliminary results}

Let us recall some properties of the capacity of general dimension $n$ (see [21] and [22]).

Proposition 3.1 Let $\Omega$ be an open subset of $\mathbf{R}^{n}$ and $E, E_{1}, E_{2}$ subsets of $\Omega$. Then

(i) $\operatorname{cap}(\emptyset, \Omega)=0$;

(ii) $E_{1} \subset E_{2} \Longrightarrow \operatorname{cap}\left(E_{1}, \Omega\right) \leq \operatorname{cap}\left(E_{2}, \Omega\right)$ (monotonicity);

(iii) $\operatorname{cap}\left(E_{1} \cup E_{2}, \Omega\right)+\operatorname{cap}\left(E_{1} \cap E_{2}, \Omega\right) \leq \operatorname{cap}\left(E_{1}, \Omega\right)+\operatorname{cap}\left(E_{2}, \Omega\right)$ (strong subadditivity);

(iv) if $\left\{E_{h}\right\}_{h}$ is an increasing sequence of subsets of $\Omega$ and $E=\bigcup_{h} E_{h} \subset \Omega$, then $\operatorname{cap}(E, \Omega)=\lim _{h} \operatorname{cap}\left(E_{h}, \Omega\right)$;

(v) if $\left\{E_{h}\right\}_{h}$ is a sequence of subsets of $\Omega$ and $E \subseteq \bigcup_{h} E_{h}$, then $\operatorname{cap}(E, \Omega) \leq \sum_{h} \operatorname{cap}\left(E_{h}, \Omega\right)$

(vi) if $\Omega_{1}$ and $\Omega_{2}$ are open subsets of $\mathbf{R}^{n}$ and $E \subset \Omega_{1} \subset \Omega_{2}$, then $\operatorname{cap}\left(E, \Omega_{2}\right) \leq \operatorname{cap}\left(E, \Omega_{1}\right)$;

(vii) if $\left\{\Omega_{h}\right\}_{h}$ is an increasing sequence of open sets such that $\bigcup_{h \in \mathbf{N}} \Omega_{h}=\Omega$, then $\lim _{h} \operatorname{cap}\left(E, \Omega_{h}\right)=\operatorname{cap}(E, \Omega)$; 
(viii) if $t>0$, then $\operatorname{cap}(t E, t \Omega)=t^{n-2} \operatorname{cap}(E, \Omega)$;

(ix) if $\left\{E_{h}\right\}_{h}$ is a decreasing sequence of compact subsets of $\Omega$ with $E=\bigcap_{h} E_{h}$, then $\operatorname{cap}(E, \Omega)=\lim _{h \rightarrow \infty} \operatorname{cap}\left(E_{h}, \Omega\right)$.

Now we recall two results of [5].

Lemma 3.1 Let $R$ a cube in $\mathbf{R}^{n}$ and $C \subset \subset R$ be a compact set with Lipschitz boundary $\partial C$ and $1 \leq p<\infty$. Then there exists a linear bounded extension operator $\Phi: W^{1, p}(R \backslash C) \rightarrow$ $W^{1, p}(R)$ such that

$$
\|\nabla(\Phi v)\|_{L^{P}(R)} \leq c\|\nabla v\|_{L^{P}(R \backslash C)}, \quad \text { for every } v \in W^{1, p}(R \backslash C)
$$

Let $T^{\prime}=Q \backslash(l \stackrel{\circ}{Y}), T_{\varepsilon}^{\prime}=\bigcup\left\{\varepsilon T^{\prime}+\varepsilon \mathbf{k}, \mathbf{k} \in \mathbf{Z}^{n}\right.$ s.t. $\left.\varepsilon(Y+\mathbf{k}) \subset \Omega\right\}$ and $\Omega_{\varepsilon}^{\prime}=\Omega \backslash T_{\varepsilon}^{\prime}$.

Theorem 3.1 Let $\Omega \subset R^{n}$ be a bounded open set, $\varepsilon>0$, and let $Y, l, Q, Y_{\varepsilon}, T_{\varepsilon}, \Omega_{\varepsilon}$ be defined as in Section 2.

Then there exists a family $\left\{P_{\varepsilon}\right\}_{\varepsilon}$ of uniform extension operators (i.e. $P_{\varepsilon} u=u_{\varepsilon}$ in $\Omega_{\varepsilon}$ ) from $H^{1}\left(\Omega_{\varepsilon}\right)$ to $H^{1}(\Omega)$, such that

$$
\begin{aligned}
& \left\|\nabla\left(P_{\varepsilon} u\right)\right\|_{L^{2}(\Omega)} \leq c\|\nabla u\|_{L^{2}\left(\Omega_{\varepsilon}\right)}, \quad \text { for every } u \in H^{1}\left(\Omega_{\varepsilon}\right), \\
& P_{\varepsilon} u\left(\varepsilon\left(x_{1}, x_{2}, \ldots, x_{n}\right)+\varepsilon \mathbf{k}\right)=P_{\varepsilon} u\left(\varepsilon\left(-x_{1}, x_{2}, \ldots, x_{n}\right)+\varepsilon \mathbf{k}\right),
\end{aligned}
$$

for every $u \in H^{1}\left(\Omega_{\varepsilon}\right)$ and for every $\left(x_{1}, x_{2}, \ldots, x_{n}\right) \in l Y$ and $\mathbf{k}$ such that $\varepsilon Y+\varepsilon \mathbf{k} \subset \Omega$. Eventually if $u=0$ on $\partial \Omega$, then $P_{\varepsilon} u=0$ on $\partial \Omega$.

As a consequence we get the following result.

Corollary 3.1 Let $\Omega \subset \mathbf{R}^{n}$ a bounded open set, $\varepsilon>0$ and let $Y, l, Q, Y_{\varepsilon}, Q_{\varepsilon}, T_{\varepsilon}, \Omega_{\varepsilon}^{\tau, \sigma}$ be defined as in Section 2.

Let $\left\{u_{\varepsilon}^{\tau, \sigma}\right\}_{\varepsilon} \subset\left(H^{1}\left(\Omega_{\varepsilon}^{\tau, \sigma}\right)\right)^{2}$ such that $u_{\varepsilon}^{\tau, \sigma}=0$ on $\Gamma_{\varepsilon}^{D, \tau, \sigma}$.

Then there exists a sequence $\left\{v_{\varepsilon}^{\tau, \sigma}\right\}_{\varepsilon} \subset\left(H^{1}(\Omega)\right)^{2}$ of uniform extensions of $\left\{u_{\varepsilon}^{\tau, \sigma}\right\}_{\varepsilon}$ and a constant $c$ independent of $\varepsilon$ such that

$$
\begin{aligned}
& v_{\varepsilon}^{\tau, \sigma}=u_{\varepsilon}^{\tau, \sigma}, \quad \text { in } \Omega_{\varepsilon}^{\tau, \sigma}, \\
& v_{\varepsilon}^{\tau, \sigma}=0, \quad \text { on } \Gamma_{\varepsilon}^{D, \tau, \sigma}, \\
& \left\|\nabla v_{\varepsilon}^{\tau, \sigma}\right\|_{L^{2}(\Omega)} \leq c\left\|\nabla u_{\varepsilon}^{\tau, \sigma}\right\|_{L^{2}\left(\Omega_{\varepsilon}^{\tau, \sigma}\right)},
\end{aligned}
$$

and

$$
v_{\varepsilon}^{\tau, \sigma}\left(x_{1}, x_{2}, \ldots, x_{n}\right)=v_{\varepsilon}^{\tau, \sigma}\left(2 \varepsilon k_{1}-x_{1}, x_{2}, \ldots, x_{n}\right)
$$

for a.e. $x \in\left(\left\{\varepsilon(l Y+\mathbf{k}), \mathbf{k} \in \mathbf{Z}^{n}\right.\right.$ s.t. $\left.\left.\varepsilon(Y+\mathbf{k}) \subset \Omega\right\} \backslash \Omega_{\varepsilon}\right) \cap \Omega_{\varepsilon}^{\prime}$ and for every $\mathbf{k}$ of this kind and related to $x$. 
Proof Let $u_{\varepsilon}^{\tau, \sigma}=\left(u_{\varepsilon, 1}^{\tau, \sigma,}, u_{\varepsilon, 2}^{\tau, \sigma}\right) \in\left(H^{1}\left(\Omega_{\varepsilon}^{\tau, \sigma}\right)\right)^{2}$ and let us define

$$
\bar{u}_{\varepsilon}^{\tau, \sigma}= \begin{cases}u_{\varepsilon}^{\tau, \sigma}, & \text { in } \Omega_{\varepsilon}^{\tau, \sigma} \\ 0, & \text { in } \Omega_{\varepsilon} \backslash \Omega_{\varepsilon}^{\tau, \sigma}\end{cases}
$$

We observe that $\partial \Omega_{\varepsilon}^{\tau, \sigma} \cap \partial\left(\Omega_{\varepsilon} \backslash \Omega_{\varepsilon}^{\tau, \sigma}\right)=\Gamma_{\varepsilon}^{D, \tau, \sigma}$ and therefore $\bar{u}_{\varepsilon}^{\tau, \sigma} \in\left(H^{1}\left(\Omega_{\varepsilon}\right)\right)^{2}$. Then the sequence $\left\{v_{\varepsilon}^{\tau, \sigma}\right\}_{\varepsilon}$ given by $v_{\varepsilon}^{\tau, \sigma}=\left(P_{\varepsilon} \bar{u}_{\varepsilon, 1}^{\tau, \sigma}, P_{\varepsilon} \bar{u}_{\varepsilon, 2}^{\tau, \sigma}\right)$ meets the requirements by Theorem 3.1 .

Let $\left(S_{\varepsilon}^{\tau, \sigma}\right)^{*}=\left\{x \in \mathbf{R}^{n}:\left(-x_{1}, x_{2}, \ldots, x_{n}\right) \in S_{\varepsilon}^{\tau, \sigma}\right\}, S_{\varepsilon}^{\prime \tau, \sigma}=S_{\varepsilon}^{\tau, \sigma} \cup\left(S_{\varepsilon}^{\tau, \sigma}\right)^{*}$ and $D_{\varepsilon}^{\prime \tau, \sigma}=\left(\bigcup\left\{S_{\varepsilon}^{\prime \tau, \sigma}+\right.\right.$ $\left.\left.\varepsilon \mathbf{k}: \mathbf{k} \in \mathbf{Z}^{n}\right\}\right) \cap Y_{\varepsilon}$ if $0<\varepsilon<\varepsilon_{\tau}, D_{\varepsilon}^{\prime \tau, \sigma}=\emptyset$ otherwise.

Let $B \subset \subset Y$ an open ball centered at the origin, $1<v<\frac{n}{n-2}(1<v<+\infty$, if $n=2)$ and construct the following families of sets:

$$
A_{\varepsilon}^{\sigma}= \begin{cases}\left(\bigcup\left\{\varepsilon^{v} B+\varepsilon \mathbf{k}: \mathbf{k} \in \mathbf{Z}^{n}\right\}\right) \cap Y_{\varepsilon}, & \text { if } \sigma \geq \frac{n}{n-2}(n \geq 3), \\ \left(\bigcup\left\{\varepsilon^{\sigma}((B+\tau) \cup(B-\tau))\right\}: \mathbf{k} \in \mathbf{Z}^{n}\right) \cap Y_{\varepsilon}, & \text { if } 1 \leq \sigma<\frac{n}{n-2}(n \geq 3) \\ \left(\bigcup\left\{\exp \left(-\frac{1}{\varepsilon^{\sigma}}\right)((B+\tau) \cup(B-\tau))\right\}: \mathbf{k} \in \mathbf{Z}^{n}\right) \cap Y_{\varepsilon}, & \text { if } \sigma<2(n=2), \\ \left(\bigcup\left\{\exp \left(-\frac{1}{\varepsilon^{\alpha}}\right) B+\varepsilon \mathbf{k}\right\}: \mathbf{k} \in \mathbf{Z}^{n}\right) \cap Y_{\varepsilon}, & \text { with } \alpha<2 \text { if } \sigma \geq 2(n=2)\end{cases}
$$

if $0<\varepsilon<\varepsilon_{1}$ and $A_{\varepsilon}^{\sigma}=\emptyset$ otherwise (let us observe that there exists $\varepsilon_{1}>0$ such that if $0<\varepsilon<$ $\varepsilon_{1}$ then $\left.S_{\varepsilon}^{\prime \tau, \sigma} \subset \subset B_{\varepsilon} \subset \subset \varepsilon Y\right)$.

Let $C_{0}^{\infty}\left(\Omega, D_{\varepsilon}^{\prime \tau \sigma}, 1\right)$ be the set of functions $v \in C_{0}^{\infty}(\Omega)$ such that $v=1$ in a neighborhood of $D_{\varepsilon}^{\prime \tau, \sigma}$.

Let $H_{0}^{1}\left(\Omega, D_{\varepsilon}^{\prime \tau, \sigma}, 1\right)$ be the closure of $C_{0}^{\infty}\left(\Omega, D_{\varepsilon}^{\prime \tau, \sigma}, 1\right)$ in $H_{0}^{1}(\Omega)$. Let $\Psi_{\varepsilon}^{\tau, \sigma}$ be the unique solution of the problem

$$
\min \left\{\int_{\Omega}|\nabla \varphi|^{2} d x: \varphi \in H_{0}^{1}\left(\Omega, D_{\varepsilon}^{\prime \tau, \sigma}, 1\right), \varphi=0 \text { on } \Omega \backslash A_{\varepsilon}^{\sigma}\right\}
$$

Let us pose $\psi_{\varepsilon}^{\tau, \sigma}=1-\Psi_{\varepsilon}^{\tau, \sigma}$. We can consider $\psi_{\varepsilon}^{\tau, \sigma}$ as a function of $H_{0}^{1}(\Omega)$, and observe that

$$
\begin{aligned}
& \psi_{\varepsilon}^{\tau, \sigma}=0, \quad \text { on } D_{\varepsilon}^{\prime \tau, \sigma}, \\
& \psi_{\varepsilon}^{\tau, \sigma}=1, \quad \text { in } \Omega \backslash A_{\varepsilon}^{\sigma} .
\end{aligned}
$$

Using a result proved in Lemma 3.5 of [5], we can study the behavior, and explicitly calculate, the 'strange term' $\mu^{\tau, \sigma}$, when $\sigma$ is equal to $\frac{n}{n-2}$, and $\sigma$ is different from $\frac{n}{n-2}$, i.e., when the distance between the small hole and the big one is different from the size of the latter. In fact if $1 \leq \sigma<\frac{n}{n-2}$ the distance between the small hole and the big one is bigger and bigger than the size of the latter; if $\sigma>\frac{n}{n-2}$ the condition is just the opposite.

Theorem 3.2 For any fixed $\varepsilon$ let $\psi_{\varepsilon}^{\tau, \sigma}$ the unique solution of (3.6). Then

$$
\begin{aligned}
& 0 \leq \psi_{\varepsilon}^{\tau, \sigma} \leq 1, \\
& \psi_{\varepsilon}^{\tau, \sigma} \rightarrow 1 \text { weakly in } H^{1}(\Omega),
\end{aligned}
$$




$$
\psi_{\varepsilon}^{\tau, \sigma} \rightarrow 1 \quad \text { strongly in } L^{p}(\Omega), \forall p \in[1,+\infty[.
$$

Moreover, for $v \geq \frac{2}{2-p}$, we have

$$
\nabla \psi_{\varepsilon}^{\tau, \sigma} \rightarrow 0 \quad \text { strongly in } L^{p}(\Omega), \forall p \in[1,2[.
$$

Furthermore, if we restrict $\psi_{\varepsilon}^{\tau, \sigma}$ to $\Omega_{\varepsilon}$, we get

$$
\psi_{\varepsilon}^{\tau, \sigma}\left(x_{1}, x_{2}, \ldots, x_{n}\right)=\psi_{\varepsilon}^{\tau, \sigma}\left(2 \varepsilon k_{1}-x_{1}, x_{2}, \ldots, x_{n}\right)
$$

for a.e. $x \in\left(\left\{\varepsilon(l Y+\mathbf{k}): \mathbf{k} \in \mathbf{Z}^{n}\right.\right.$ s.t. $\left.\left.\varepsilon(Y+\mathbf{k}) \subset \Omega\right\} \backslash \Omega_{\varepsilon}\right) \cap \Omega_{\varepsilon}^{\prime}$ and for every $\mathbf{k}$ of this kind and related to $x$.

Moreover, there exist $\mu_{\varepsilon}^{\tau, \sigma}$ and $\gamma_{\varepsilon}^{\tau, \sigma} \in H^{-1}(\Omega)$ and a unique distribution $\mu^{\tau, \sigma} \in W^{-1, \infty}$ such that

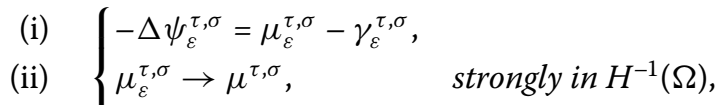

$$
\begin{aligned}
& \text { (iii) } \quad\left\langle\gamma_{\varepsilon}^{\tau, \sigma}, v_{\varepsilon}\right\rangle=0, \quad \forall v_{\varepsilon} \in H_{0}^{1}\left(\Omega \backslash D_{\varepsilon}^{\prime \tau, \sigma}\right) \text {, }
\end{aligned}
$$

where

$$
\mu^{\tau, \sigma}= \begin{cases}2 \operatorname{cap}(K), & \text { if } 1 \leq \sigma<\frac{n}{n-2} \text { for every } \tau \\ \operatorname{cap}\left((K+\tau) \cup\left(K^{*}-\tau\right)\right), & \text { if } \sigma=\frac{n}{n-2}, \\ \operatorname{cap}\left(K \cup K^{*}\right), & \text { if } \sigma>\frac{n}{n-2} \text { for every } \tau,\end{cases}
$$

if $n \geq 3$, and

$$
\mu^{\tau, \sigma}= \begin{cases}4 \pi, & \text { if } \sigma<2, \\ 2 \pi, & \text { if } \sigma \geq 2,\end{cases}
$$

if $n=2$.

Proof Properties (3.8)-(3.10) are proved in [3].

By arguing as in Lemma 3.5 of [5] we obtain conditions (3.11) and (3.12). Moreover, Theorem 2.7 and Lemma 2.8 of [3] provide the existence of two sequences $\left\{\mu_{\varepsilon}^{\tau, \sigma}\right\}_{\varepsilon},\left\{\gamma_{\varepsilon}^{\tau, \sigma}\right\}_{\varepsilon} \in$ $H^{-1}(\Omega)$ and $\mu^{\tau, \sigma} \in W^{-1, \infty}$ such that (3.13) holds up to a subsequence.

Now we identify the measure $\mu^{\tau, \sigma}$.

Let us recall that by virtue of Proposition 1.1 in [3] up to a subsequence

$$
\left\langle\mu_{\varepsilon}^{\tau, \sigma}, \varphi\right\rangle=\lim _{\varepsilon} \int\left|\nabla \psi_{\varepsilon}^{\tau, \sigma}\right|^{2} \varphi, \quad \forall \varphi \in \mathcal{D}(\Omega) .
$$

The proof of (3.14) and (3.15) will be performed in five steps. 
Step 1. Let $n \geq 3$ and $\sigma=\frac{n}{n-2}$. At first we observe that

$$
\begin{aligned}
\left\langle-\Delta \psi_{\varepsilon}^{\tau, \sigma}, \varphi \psi_{\varepsilon}^{\tau, \sigma}\right\rangle & =\int_{\Omega} \nabla \psi_{\varepsilon}^{\tau, \sigma} \nabla\left(\varphi \psi_{\varepsilon}^{\tau, \sigma}\right) d x \\
& =\int_{\Omega} \nabla \psi_{\varepsilon}^{\tau, \sigma}(\nabla \varphi) \psi_{\varepsilon}^{\tau, \sigma} d x+\int_{\Omega} \nabla \psi_{\varepsilon}^{\tau, \sigma}\left(\nabla \psi_{\varepsilon}^{\tau, \sigma}\right) \varphi d x \\
& =\int_{\Omega} \nabla \psi_{\varepsilon}^{\tau, \sigma}(\nabla \varphi) \psi_{\varepsilon}^{\tau, \sigma} d x+\int_{\Omega}\left|\nabla \psi_{\varepsilon}^{\tau, \sigma}\right|^{2} \varphi
\end{aligned}
$$

Let us consider the first term in the right-hand side of (3.16); by (3.10) and (3.11), one has

$$
\lim _{\varepsilon \rightarrow 0} \int_{\Omega} \nabla \psi_{\varepsilon}^{\tau, \sigma}(\nabla \varphi) \psi_{\varepsilon}^{\tau, \sigma} d x=0, \quad \forall \varphi \in \mathcal{D}(\Omega)
$$

Let us consider $\varphi \in \mathcal{D}(\Omega)$. Let us fix $\delta>0$ and consider $\eta>0$ such that

$$
|x-y|<\eta \quad \Longrightarrow \quad|\varphi(x)-\varphi(y)|<\delta \text {. }
$$

Then if $\varepsilon>0$ is small enough

$$
\begin{aligned}
& \varepsilon^{-n} \int_{\varepsilon Y+\varepsilon \mathbf{k}}(\varphi(y)-\delta) d y \int_{\varepsilon Y+\varepsilon \mathbf{k}}\left|\nabla \psi_{\varepsilon}^{\tau, \sigma}\right|^{2} d x \\
& \quad \leq \int_{\varepsilon Y+\varepsilon \mathbf{k}}\left|\nabla \psi_{\varepsilon}^{\tau, \sigma}\right|^{2} \varphi d x \\
& \quad \leq \varepsilon^{-n} \int_{\varepsilon Y+\varepsilon \mathbf{k}}(\varphi(y)+\delta) d y \int_{\varepsilon Y+\varepsilon \mathbf{k}}\left|\nabla \psi_{\varepsilon}^{\tau, \sigma}\right|^{2} d x
\end{aligned}
$$

for every $\mathbf{k} \in \mathbf{Z}^{n}$ such that $\varepsilon Y+\varepsilon \mathbf{k} \subset \Omega$.

Moreover, by definition of $\psi_{\varepsilon}^{\tau, \sigma}$, taking into account its $\varepsilon Y$ periodicity in $Y_{\varepsilon}$ and by (viii) of Proposition 3.1, if $n \geq 3$, we have

$$
\begin{aligned}
\int_{\varepsilon Y+\varepsilon \mathbf{k}}\left|\nabla \psi_{\varepsilon}^{\tau, \sigma}\right|^{2} & =\int_{\varepsilon Y+\varepsilon \mathbf{k}}\left|\nabla \Psi_{\varepsilon}^{\tau, \sigma}\right|^{2}=\int_{\varepsilon^{\nu} B+\varepsilon \mathbf{k}}\left|\nabla \Psi_{\varepsilon}^{\tau, \sigma}\right|^{2} \\
& =\operatorname{cap}\left(\varepsilon^{\frac{n}{n-2}}\left((K+\tau) \cup\left(K^{*}-\tau\right)\right), \varepsilon^{\nu} B\right) \\
& =\varepsilon^{n} \operatorname{cap}\left((K+\tau) \cup\left(K^{*}-\tau\right), \varepsilon^{\nu-\frac{n}{n-2}} B\right) .
\end{aligned}
$$

Now if $\varepsilon>0$ is small enough, then $\operatorname{supp}(\varphi) \subset Y_{\varepsilon}$ and by the last expression and summing up relations (3.18), for every $\mathbf{k} \in \mathbf{Z}^{n}$ such that $\varepsilon Y+\varepsilon \mathbf{k} \subset \Omega$, we have

$$
\begin{aligned}
& \operatorname{cap}\left((K+\tau) \cup\left(K^{*}-\tau\right), \varepsilon^{\nu-\frac{n}{n-2}} B\right) \int_{\Omega}(\varphi(y)-\delta) \\
& \quad \leq \int_{\Omega}\left|\nabla \psi_{\varepsilon}^{\tau, \sigma}\right|^{2} \varphi \\
& \quad \leq \operatorname{cap}\left((K+\tau) \cup\left(K^{*}-\tau\right), \varepsilon^{\nu-\frac{n}{n-2}} B\right) \int_{\Omega}(\varphi(y)+\delta), \quad \forall \varphi \in \mathcal{D}(\Omega) .
\end{aligned}
$$


Then by (vii) of Proposition 3.1 and the arbitrariness of $\delta$, passing to the limit for $\varepsilon \rightarrow 0$, we obtain

$$
\lim _{\varepsilon \rightarrow 0} \int_{\Omega}\left|\nabla \psi_{\varepsilon}^{\tau, \sigma}\right|^{2} \varphi=\operatorname{cap}\left((K+\tau) \cup\left(K^{*}-\tau\right)\right) \int_{\Omega} \varphi(x) d x, \quad \forall \varphi \in \mathcal{D}(\Omega) .
$$

Indeed, using condition (i) in (3.13), one has

$$
\begin{aligned}
\left\langle\Delta \psi_{\varepsilon}^{\tau, \sigma}, \varphi \psi_{\varepsilon}^{\tau, \sigma}\right\rangle & =\left\langle\mu_{\varepsilon}^{\tau, \sigma}-\gamma_{\varepsilon}^{\tau, \sigma}, \varphi \psi_{\varepsilon}^{\tau, \sigma}\right\rangle \\
& =\left\langle\mu_{\varepsilon}^{\tau, \sigma}, \varphi \psi_{\varepsilon}^{\tau, \sigma}\right\rangle-\left\langle\gamma_{\varepsilon}^{\tau, \sigma}, \varphi \psi_{\varepsilon}^{\tau, \sigma}\right\rangle, \quad \forall \varphi \in \mathcal{D}(\Omega) .
\end{aligned}
$$

Consequently by combining (3.13)(iii), with (3.16), (3.17), and (3.21), we obtain

$$
\left\langle\mu^{\tau, \sigma}, \varphi\right\rangle=\operatorname{cap}\left((K+\tau) \cup\left(K^{*}-\tau\right)\right) \int_{\Omega} \varphi(x) d x, \quad \forall \varphi \in \mathcal{D}(\Omega) .
$$

Step 2. Let $n \geq 3$ and let us consider the case $\sigma>\frac{n}{n-2}$. We set

$$
\begin{aligned}
& K^{\prime}=K \cup K^{*}, \\
& K_{\varepsilon}^{\gamma}=\left(\left(K+\varepsilon^{\gamma} \tau\right) \cup\left(K^{*}-\varepsilon^{\gamma} \tau\right)\right), \\
& \left(K^{\prime}\right)_{\delta}=\left\{x \in \mathbf{R}^{n}: \operatorname{dist}\left(x, K^{\prime}\right)<\delta\right\},
\end{aligned}
$$

where $\gamma=\sigma-\frac{n}{n-2}$. As in the previous case

$$
\begin{aligned}
\int_{\varepsilon Y+\varepsilon \mathbf{k}}\left|\nabla \psi_{\varepsilon}^{\tau, \sigma}\right|^{2} & =\int_{\varepsilon^{\nu} B+\varepsilon \tau}\left|\nabla \Psi_{\varepsilon}^{\tau, \sigma}\right|^{2} \\
& =\operatorname{cap}\left(\left(\varepsilon^{\frac{n}{n-2}} K+\varepsilon^{\sigma} \tau\right) \cup\left(\varepsilon^{\frac{n}{n-2}} K^{*}-\varepsilon^{\sigma} \tau\right), \varepsilon^{\nu} B\right) \\
& =\varepsilon^{n} \operatorname{cap}\left(\left(K+\varepsilon^{\gamma} \tau\right) \cup\left(K^{*}-\varepsilon^{\gamma} \tau\right), \varepsilon^{\nu-\frac{n}{n-2}} B\right)=\varepsilon^{n} \operatorname{cap}\left(K_{\varepsilon}^{\gamma}, \varepsilon^{\nu-\frac{n}{n-2}} B\right) .
\end{aligned}
$$

Let us fix $\delta \in] 0,1\left[\right.$, and $r>\operatorname{dist}\left(K^{\prime}\right)+1$. If $\varepsilon$ is small enough, we have $K_{\varepsilon}^{\gamma} \subseteq\left(K^{\prime}\right)_{\delta}$ and $r B \subseteq$ $\varepsilon^{\nu-\frac{n}{n-2}} B$. Then

$$
\operatorname{cap}\left(K_{\varepsilon}^{\gamma}, \varepsilon^{\nu-\frac{n}{n-2}} B\right) \leq \operatorname{cap}\left(\left(K^{\prime}\right)_{\delta}, \varepsilon^{\nu-\frac{n}{n-2}} B\right) \leq \operatorname{cap}\left(\left(K^{\prime}\right)_{\delta}, r B\right),
$$

for $\varepsilon$ is small enough (dependent on $r$ ).

\section{Consequently}

$$
\limsup _{\varepsilon \rightarrow 0} \operatorname{cap}\left(K_{\varepsilon}^{\gamma}, \varepsilon^{\nu-\frac{n}{n-2}} B\right) \leq \operatorname{cap}\left(\left(K^{\prime}\right)_{\delta}, r B\right) .
$$

On the other hand

$$
\operatorname{cap}\left(K_{\varepsilon}^{\gamma}, \varepsilon^{\nu-\frac{n}{n-2}} B\right) \geq \operatorname{cap}\left(K_{\varepsilon}^{\gamma} \cap K^{\prime}, \varepsilon^{\nu-\frac{n}{n-2}} B\right) \geq \operatorname{cap}\left(K_{\varepsilon}^{\gamma} \cap K^{\prime}\right), \quad \forall \varepsilon>0 .
$$

Let us observe that $K_{\varepsilon}^{\gamma} \cap K^{\prime}$ is an increasing sequence converging to $K^{\prime}$, and $K_{\varepsilon}^{\gamma} \cap K^{\prime} \neq \emptyset$ since $K$ is a perfect set. Consequently, by (iv) of Proposition 3.1, it follows that

$$
\lim _{\varepsilon \rightarrow 0^{+}} \operatorname{cap}\left(K_{\varepsilon}^{\gamma} \cap K^{\prime}\right)=\operatorname{cap}\left(K^{\prime}\right) .
$$


Therefore by combining (3.24) with (3.25) and (3.26) we get

$$
\begin{aligned}
\operatorname{cap}\left(K^{\prime}\right) & =\lim _{\varepsilon \rightarrow 0^{+}} \operatorname{cap}\left(K_{\varepsilon}^{\gamma} \cap K^{\prime}\right) \leq \liminf _{\varepsilon \rightarrow 0^{+}} \operatorname{cap}\left(K_{\varepsilon}^{\gamma}, \varepsilon^{\nu-\frac{n}{n-2}} B\right) \\
& \leq \limsup _{\varepsilon \rightarrow 0^{+}} \operatorname{cap}\left(K_{\varepsilon}^{\gamma}, \varepsilon^{\nu-\frac{n}{n-2}} B\right) \leq \operatorname{cap}\left(\left(K^{\prime}\right)_{\delta}, r B\right) .
\end{aligned}
$$

Since $K$ is a compact set, $\left(K^{\prime}\right)_{\delta}$ decreases to $K^{\prime}$ as $\delta \rightarrow 0$. Then, by (ix) of Proposition 3.1, one has

$$
\lim _{\delta \rightarrow 0^{+}} \operatorname{cap}\left(\left(K^{\prime}\right)_{\delta}, r B\right)=\operatorname{cap}\left(K^{\prime}, r B\right)
$$

By passing to the limit, as $\delta \rightarrow 0^{+}$in (3.27), by (3.28), it follows that

$$
\operatorname{cap}\left(K^{\prime}\right) \leq \liminf _{\varepsilon \rightarrow 0^{+}} \operatorname{cap}\left(K_{\varepsilon}^{\gamma}, \varepsilon^{\nu-\frac{n}{n-2}} B\right) \leq \limsup _{\varepsilon \rightarrow 0^{+}} \operatorname{cap}\left(K_{\varepsilon}^{\gamma}, \varepsilon^{\nu-\frac{n}{n-2}} B\right) \leq \operatorname{cap}\left(K^{\prime}, r B\right) .
$$

By passing to the limit as $r \rightarrow \infty$ in (3.29) we have the result

$$
\lim _{\varepsilon \rightarrow 0^{+}} \operatorname{cap}\left(K_{\varepsilon}^{\gamma}, \varepsilon^{\nu-\frac{n}{n-2}} B\right)=\operatorname{cap}\left(K^{\prime}\right)
$$

By arguing as in the previous case, (3.30) provides

$$
\left\langle\mu^{\tau, \sigma}, \varphi\right\rangle=\operatorname{cap}\left(K^{\prime}\right) \int_{\Omega} \varphi(x) d x, \quad \forall \varphi \in \mathcal{D}(\Omega) .
$$

Step 3. Let $n \geq 3$ and let us examine the case $1 \leq \sigma<\frac{n}{n-2}$.

By recalling the expression of the $A_{\varepsilon}^{\sigma}$ in (3.5), and by following the same arguments as above, one has

$$
\begin{aligned}
\int_{\varepsilon Y+\varepsilon \mathbf{k}}\left|\nabla \psi_{\varepsilon}^{\tau, \sigma}\right|^{2} & =\int_{\varepsilon^{\sigma}(B+\tau) \cup \varepsilon^{\sigma}(B-\tau)}\left|\nabla \Psi_{\varepsilon}^{\tau, \sigma}\right|^{2}=2 \int_{\varepsilon^{\sigma}(B+\tau)}\left|\nabla \Psi_{\varepsilon}^{\tau, \sigma}\right|^{2} \\
& =2 \operatorname{cap}\left(\left(\varepsilon^{\frac{n}{n-2}} K+\varepsilon^{\sigma} \tau\right), \varepsilon^{\sigma}(B+\tau)\right)=2 \operatorname{cap}\left(\varepsilon^{\frac{n}{n-2}} K, \varepsilon^{\sigma} B\right) \\
& =2 \varepsilon^{n} \operatorname{cap}\left(K, \varepsilon^{\sigma-\frac{n}{n-2}} B\right) .
\end{aligned}
$$

By repeating the same steps (3.16)-(3.22), we have

$$
\left\langle\mu^{\tau, \sigma}, \varphi\right\rangle=2 \operatorname{cap}(K) \int_{\Omega} \varphi(x) d x, \quad \forall \varphi \in \mathcal{D}(\Omega) .
$$

Step 4. Let $n=2$. Let $\sigma<2$, and $A_{\varepsilon}^{\sigma}$ be the respective family sets (see (3.5)). In a similar way as in the previous case, by (viii) of Proposition 3.1 we have

$$
\begin{aligned}
\int_{\varepsilon Y+\varepsilon \mathbf{k}}\left|\nabla \psi_{\varepsilon}^{\tau, \sigma}\right|^{2} d x & =\int_{\exp \left(\varepsilon^{-\sigma}\right)((B+\tau) \cup(B-\tau))}\left|\nabla \Psi_{\varepsilon}^{\tau, \sigma}\right|^{2} \\
& =2 \int_{\exp \left(\varepsilon^{-\sigma}\right)((B+\tau))}\left|\nabla \Psi_{\varepsilon}^{\tau, \sigma}\right|^{2}=2 \operatorname{cap}\left(\exp \left(-\frac{1}{\varepsilon^{2}}\right) K, \exp \left(-\frac{1}{\varepsilon^{\sigma}}\right) B\right) \\
& =2 \operatorname{cap}\left(K, \exp \left(-\frac{1}{\varepsilon^{\sigma}}+\frac{1}{\varepsilon^{2}}\right) B\right)
\end{aligned}
$$


Let us observe that $E=K \cap\left\{x \in \mathbf{R}^{n}: x_{1}=0\right\}$ contains a segment and that $K$ is well contained in another ball centered at the origin, say it $B_{1}$. So, by (ii) of Proposition 3.1 we have

$$
\begin{aligned}
\frac{1}{\varepsilon^{2}} \operatorname{cap}\left(E, \exp \left(-\frac{1}{\varepsilon^{\sigma}}+\frac{1}{\varepsilon^{2}}\right) B\right) & \leq \frac{1}{\varepsilon^{2}} \operatorname{cap}\left(K, \exp \left(-\frac{1}{\varepsilon^{\sigma}}+\frac{1}{\varepsilon^{2}}\right) B\right) \\
& \leq \frac{1}{\varepsilon^{2}} \operatorname{cap}\left(B_{1}, \exp \left(-\frac{1}{\varepsilon^{\sigma}}+\frac{1}{\varepsilon^{2}}\right) B\right)
\end{aligned}
$$

By Lemma 3.3 of [10] we obtain

$$
\begin{aligned}
\frac{1}{\varepsilon^{2}} \frac{2 \pi}{-\varepsilon^{-\sigma}+\varepsilon^{-2}}\left(1+b_{\varepsilon}\right) & \leq \frac{1}{\varepsilon^{2}} \operatorname{cap}\left(K, \exp \left(-\frac{1}{\varepsilon^{\sigma}}+\frac{1}{\varepsilon^{2}}\right) B\right) \\
& \leq \frac{1}{\varepsilon^{2}} \frac{2 \pi}{-\varepsilon^{-\sigma}+\varepsilon^{-2}}\left(1+a_{\varepsilon}\right),
\end{aligned}
$$

where

$$
\lim _{\varepsilon \rightarrow 0} b_{\varepsilon}=0 \quad \text { and } \quad \lim _{\varepsilon \rightarrow 0} a_{\varepsilon}=0,
$$

so that

$$
\frac{1}{\varepsilon^{2}} \operatorname{cap}\left(K, \exp \left(-\frac{1}{\varepsilon^{\sigma}}+\frac{1}{\varepsilon^{2}}\right) B\right) \rightarrow 2 \pi .
$$

Hence by (3.18) and (3.34) one has

$$
\lim _{\varepsilon \rightarrow 0} \int_{\Omega}\left|\nabla \psi_{\varepsilon}^{\tau, \sigma}\right|^{2} \varphi d x=4 \pi \int_{\Omega} \varphi(x) d x
$$

Then we obtain

$$
\left\langle\mu^{\tau, \sigma}, \varphi\right\rangle=4 \pi \int_{\Omega} \varphi(x) d x, \quad \forall \varphi \in \mathcal{D}(\Omega) .
$$

Step 5. Finally let $n=2$ and let us examine the case $\sigma \geq 2$ setting

$$
K_{\varepsilon, \sigma}=\exp \left(-\frac{1}{\varepsilon^{2}}\right) K+\exp \left(-\frac{1}{\varepsilon^{\sigma}}\right) \tau
$$

and

$$
K_{\varepsilon, \sigma}^{*}=\exp \left(-\frac{1}{\varepsilon^{2}}\right) K^{*}-\exp \left(-\frac{1}{\varepsilon^{\sigma}}\right) \tau .
$$

Let us suppose that $E=K \cap\left\{x \in \mathbf{R}^{n}: x_{1}=0\right\}$ contains a segment. Let us denote by $E_{\varepsilon, \sigma}$ and $E_{\varepsilon, \sigma}^{*}$, respectively, the following subsets:

$$
\begin{aligned}
& E_{\varepsilon, \sigma}=\exp \left(-\frac{1}{\varepsilon^{2}}\right) E+\exp \left(-\frac{1}{\varepsilon^{\sigma}}\right) \tau, \\
& E_{\varepsilon, \sigma}^{*}=\exp \left(-\frac{1}{\varepsilon^{2}}\right) E^{*}-\exp \left(-\frac{1}{\varepsilon^{\sigma}}\right) \tau,
\end{aligned}
$$

where with $E^{*}$ we denote the symmetry of subset $E$ with respect to the hyperplane $\left\{x_{1}=0\right\}$. 
By (ii) and (viii) of Proposition 3.1, and by Lemma 3.3 of [10] we have

$$
\begin{aligned}
\int_{\varepsilon Y+\varepsilon \mathbf{k}}\left|\nabla \psi_{\varepsilon}^{\tau, \sigma}\right|^{2} d x= & \int_{\exp \left(\varepsilon^{-\alpha}\right)(B+\tau)}\left|\nabla \Psi_{\varepsilon}^{\tau, \sigma}\right|^{2}=\operatorname{cap}\left(K_{\varepsilon, \sigma}^{*} \cup K_{\varepsilon, \sigma}, \exp \left(-\frac{1}{\varepsilon^{\alpha}}\right) B\right) \\
= & \operatorname{cap}\left(\left(K+\exp \left(\frac{1}{\varepsilon^{2}}-\frac{1}{\varepsilon^{\sigma}}\right) \tau\right)\right. \\
& \left.\cup\left(K^{*}-\exp \left(\frac{1}{\varepsilon^{2}}-\frac{1}{\varepsilon^{\sigma}}\right) \tau\right), \exp \left(\frac{1}{\varepsilon^{2}}-\frac{1}{\varepsilon^{\alpha}}\right) B\right) .
\end{aligned}
$$

Let $B_{2}$ be another ball centered at the origin and containing

$$
\left(K+\exp \left(\frac{1}{\varepsilon^{2}}-\frac{1}{\varepsilon^{\sigma}}\right) \tau\right) \cup\left(K^{*}-\exp \left(\frac{1}{\varepsilon^{2}}-\frac{1}{\varepsilon^{\sigma}}\right) \tau\right)
$$

then on the one hand we have

$$
\begin{aligned}
& \frac{1}{\varepsilon^{2}} \operatorname{cap}\left(\left(K+\exp \left(\frac{1}{\varepsilon^{2}}-\frac{1}{\varepsilon^{\sigma}}\right) \tau\right) \cup\left(K^{*}-\exp \left(\frac{1}{\varepsilon^{2}}-\frac{1}{\varepsilon^{\sigma}}\right) \tau\right), \exp \left(\frac{1}{\varepsilon^{2}}-\frac{1}{\varepsilon^{\alpha}}\right) B\right) \\
& \leq \frac{1}{\varepsilon^{2}} \operatorname{cap}\left(B_{2}, \exp \left(\frac{1}{\varepsilon^{2}}-\frac{1}{\varepsilon^{\alpha}}\right) B\right)=\frac{1}{\varepsilon^{2}} \frac{2 \pi}{-\varepsilon^{-\alpha}+\varepsilon^{-2}}\left(1+a_{\varepsilon}\right),
\end{aligned}
$$

on the other hand

$$
\begin{aligned}
& \frac{1}{\varepsilon^{2}} \operatorname{cap}\left(K_{\varepsilon, \sigma} \cup K_{\varepsilon, \sigma}^{*}, \exp \left(-\frac{1}{\varepsilon^{\alpha}}\right) B\right) \\
& \quad \geq \frac{1}{\varepsilon^{2}} \operatorname{cap}\left(E_{\varepsilon, \sigma} \cup E_{\varepsilon, \sigma}^{*}, \exp \left(-\frac{1}{\varepsilon^{\alpha}}\right) B\right) \\
& \quad \geq \frac{1}{\varepsilon^{2}} \operatorname{cap}\left(E_{\varepsilon, \sigma}, \exp \left(-\frac{1}{\varepsilon^{\alpha}}\right) B\right) \geq \operatorname{cap}\left(\exp \left(-\frac{1}{\varepsilon^{2}}\right) E, \exp \left(-\frac{1}{\varepsilon^{\alpha}}\right) B\right) \\
& \quad=\frac{1}{\varepsilon^{2}} \operatorname{cap}\left(E, \exp \left(\frac{1}{\varepsilon^{2}}-\frac{1}{\varepsilon^{\alpha}}\right) B\right)=\frac{1}{\varepsilon^{2}} \frac{2 \pi}{-\varepsilon^{-\alpha}+\varepsilon^{-2}}\left(1+b_{\varepsilon}\right),
\end{aligned}
$$

where

$$
\lim _{\varepsilon \rightarrow 0} b_{\varepsilon}=0 \quad \text { and } \quad \lim _{\varepsilon \rightarrow 0} a_{\varepsilon}=0 .
$$

So

$$
\frac{1}{\varepsilon^{2}} \operatorname{cap}\left(K_{\varepsilon, \sigma} \cup K_{\varepsilon, \sigma}^{*}, \exp \left(-\frac{1}{\varepsilon^{\alpha}}\right) B\right) \rightarrow 2 \pi
$$

Indeed as in the previous case, by Lemma 3.3 of [10], we obtain

$$
\left\langle\mu^{\tau, \sigma}, \varphi\right\rangle=2 \pi \int_{\Omega} \varphi(x) d x, \quad \forall \varphi \in \mathcal{D}(\Omega)
$$

The following lemma is a consequence of [23] and Lemma 3.11 of [10]. 
Lemma 3.2 Let $C$ be a compact set such that $C \subset \subset Y \subset \mathbf{R}^{n}, a \geq 1$, and

$$
\Omega_{\varepsilon}^{\prime \prime}=\Omega \backslash\left(\bigcup\left\{\varepsilon^{a} C+\varepsilon \mathbf{k}: \mathbf{k} \in \mathbf{Z}^{n}\right\} \cap Y_{\varepsilon}\right)
$$

Let $\Omega \subset \mathbf{R}^{n}$ a bounded open set, $\varepsilon>0$, and let $\Omega_{\varepsilon}^{\prime \prime}$ defined as in (3.36).

Let $\left\{u_{\varepsilon}\right\}_{\varepsilon}$ and $\left\{v_{\varepsilon}\right\}_{\varepsilon}$ two bounded sequences in $\left(H_{0}^{1}(\Omega)\right)^{2}$ such that $u_{\varepsilon}=v_{\varepsilon}$ a.e. in $\Omega_{\varepsilon}^{\prime \prime}$. Then

$$
u_{\varepsilon}-v_{\varepsilon} \longrightarrow 0 \text { weakly in }\left(H_{0}^{1}(\Omega)\right)^{2} \text { as } \varepsilon \rightarrow 0^{+} .
$$

Lemma 3.3 Let $\Omega, Y, l, Q, Y_{\varepsilon}, Q_{\varepsilon}, T_{\varepsilon}, \Omega_{\varepsilon}, g_{\varepsilon}$ be defined as in Section 2 , and $\varphi=\left(\varphi_{1}, \varphi_{2}\right) a$ function in $\left(H^{1}\left(\Omega_{\varepsilon}\right)\right)^{2}$. Then there exists a constant $C$, depending on $|\Omega|$, such that for every fixed $i=1,2$

$$
\int_{\partial T_{\varepsilon}} g_{\varepsilon, i} \varphi_{i} d \mathcal{H}^{n-1} \leq C\left\|\nabla \varphi_{i}\right\|_{L^{2}\left(\Omega_{\varepsilon}\right)}
$$

Proof For every $i$ fixed, let us consider the following problem:

$$
\begin{cases}-\Delta \beta_{i}=0, & \text { in } Y^{c} \\ \frac{\partial \beta_{i}}{\partial \mathbf{n}}=g_{i}, & \text { on } \partial Q \\ \beta \text { is } Y \text {-periodic s.t. } m_{\partial Q}(\beta)=0 . & \end{cases}
$$

Set $\beta_{\varepsilon, i}(x)=\varepsilon \beta_{i}\left(\frac{x}{\varepsilon}\right)$ where $x \in Y^{c}$, so that problem (3.39) becomes

$$
\begin{cases}-\Delta \beta_{\varepsilon, i}=0, & \text { in } \Omega_{\varepsilon}, \\ \frac{\partial \beta_{\varepsilon, i}}{\partial \mathbf{n}}=g_{\varepsilon, i}, & \text { on } \partial T_{\varepsilon}\end{cases}
$$

whose variational formulation is

$$
\int_{\Omega_{\varepsilon}} \nabla \beta_{\varepsilon, i} \nabla \varphi_{i}=\int_{\partial T_{\varepsilon}} g_{\varepsilon, i} \varphi_{i} d \mathcal{H}^{n-1}, \quad \varphi_{i} \in H^{1}\left(\Omega_{\varepsilon}\right) .
$$

By Hölder's inequality we have

$$
\begin{aligned}
\int_{\partial T_{\varepsilon}} g_{\varepsilon, i} \varphi_{i} d \mathcal{H}^{n-1} & =\int_{\Omega_{\varepsilon}} \nabla \beta_{i}\left(\frac{x}{\varepsilon}\right) \nabla \varphi_{i}(x) d x \leq\left(\int_{\Omega_{\varepsilon}}\left|\nabla \beta_{i}\left(\frac{x}{\varepsilon}\right)\right|^{2} d x\right)^{\frac{1}{2}}\left\|\nabla \varphi_{i}\right\|_{L^{2}\left(\Omega_{\varepsilon}\right)} \\
& \leq\left(\frac{c|\Omega|}{\varepsilon^{2}}\right)\left(\int_{\varepsilon Y^{c}}\left|\nabla \beta_{i}\left(\frac{x}{\varepsilon}\right)\right|^{2} d x\right)^{\frac{1}{2}}\left\|\nabla \varphi_{i}\right\|_{L^{2}\left(\Omega_{\varepsilon}\right)} \\
& =c|\Omega|\left(\int_{Y^{c}}\left|\nabla \beta_{i}(y)\right|^{2} d y\right)^{\frac{1}{2}}\left\|\nabla \varphi_{i}\right\|_{L^{2}\left(\Omega_{\varepsilon}\right)}=c|\Omega|\left\|\nabla \beta_{i}\right\|_{L^{2}\left(Y^{c}\right)}\left\|\nabla \varphi_{i}\right\|_{L^{2}\left(\Omega_{\varepsilon}\right)}
\end{aligned}
$$

and the lemma is proved.

\section{Proof of Theorem 2.1}

\subsection{Compactness and convergence results}

To simplify the notation we omit the explicit dependence on the parameter $\tau$. 
We denote by $\tilde{u}$ the zero extension to the whole $\Omega$ of a vector function $u$ defined on a subset of $\Omega$ and by $\widehat{u}$ the periodic extension to $\mathbf{R}^{n} \backslash \bigcup_{k \in \mathbf{Z}^{2}}(Q+k)$ of a vector function $u$ $Y$-periodic in $H^{1}\left(Y^{c}\right)$. Moreover, let $\bar{u}_{\varepsilon}^{\sigma}$ be defined as in (3.4).

By Corollary 3.1 there exists a sequence $\left(v_{\varepsilon}^{\sigma}\right)_{\varepsilon}$ satisfying (3.2). So by Poincarés inequality and (3.2) in Corollary 3.1 we get

$$
\begin{aligned}
\left\|v_{\varepsilon}^{\sigma}\right\|_{H_{0}^{1}(\Omega)}^{2} & \leq c_{\Omega}^{2} \int_{\Omega}\left|\nabla v_{\varepsilon}^{\sigma}\right|^{2} \leq c_{\Omega}^{2} c^{2} \int_{\Omega_{\varepsilon}^{\sigma}}\left|\nabla u_{\varepsilon}^{\sigma}\right|^{2} \\
& =c_{\Omega}^{2} c^{2}\left[\int_{\Omega_{\varepsilon}^{\sigma}} f u_{\varepsilon}^{\sigma}-\int_{\Omega_{\varepsilon}^{\sigma}}\left|u_{\varepsilon}^{\sigma}\right|^{4}+\int_{\Omega_{\varepsilon}^{\sigma}}\left|u_{\varepsilon}^{\sigma}\right|^{2}+\int_{\Gamma_{\varepsilon}^{N, \sigma}} g_{\varepsilon} u_{\varepsilon}^{\sigma}\right] \\
& =c_{\Omega}^{2} c^{2}\left[\int_{\Omega_{\varepsilon}} f \bar{u}_{\varepsilon}^{\sigma}-\int_{\Omega_{\varepsilon}}\left|\bar{u}_{\varepsilon}^{\sigma}\right|^{4}+\int_{\Omega_{\varepsilon}}\left|\bar{u}_{\varepsilon}\right|^{2}+\int_{\partial T_{\varepsilon}} g_{\varepsilon} \bar{u}_{\varepsilon}^{\sigma}\right],
\end{aligned}
$$

where $c_{\Omega}$ is the Poincaré constant of $\Omega$ and $c$ is the constant given by Corollary 3.1.

By the Hölder inequality we get

$$
\left\|\bar{u}_{\varepsilon}^{\sigma}\right\|_{L^{2}\left(\Omega_{\varepsilon}\right)} \leq|\Omega|^{\frac{1}{4}}\left\|\bar{u}_{\varepsilon}^{\sigma}\right\|_{L^{4}\left(\Omega_{\varepsilon}\right)} .
$$

By Lemma 3.3 and (4.2), inequality (4.1) becomes

$$
\begin{aligned}
\left\|v_{\varepsilon}^{\sigma}\right\|_{H_{0}^{1}(\Omega)}^{2} \leq & c_{\Omega}^{2} c^{2}\left[\|f\|_{L^{2}(\Omega)}\left\|\bar{u}_{\varepsilon}^{\sigma}\right\|_{L^{2}\left(\Omega_{\varepsilon}\right)}-\left\|\bar{u}_{\varepsilon}^{\sigma}\right\|_{L^{4}\left(\Omega_{\varepsilon}\right)}^{4}\right. \\
& \left.+|\Omega|^{\frac{1}{2}}\left\|\bar{u}_{\varepsilon}^{\sigma}\right\|_{L^{4}\left(\Omega_{\varepsilon}\right)}^{2}+C\left\|\nabla \bar{u}_{\varepsilon}^{\sigma}\right\|_{L^{2}\left(\Omega_{\varepsilon}\right)}\right] .
\end{aligned}
$$

Now let us consider the following quantity:

$$
\left\|\bar{u}_{\varepsilon}^{\sigma}\right\|_{L^{4}\left(\Omega_{\varepsilon}\right)}^{4}-|\Omega|^{\frac{1}{2}}\left\|\bar{u}_{\varepsilon}^{\sigma}\right\|_{L^{4}\left(\Omega_{\varepsilon}\right)}^{2} .
$$

Let us suppose

$$
\left\|\bar{u}_{\varepsilon}^{\sigma}\right\|_{L^{4}\left(\Omega_{\varepsilon}\right)} \geq|\Omega|^{\frac{1}{4}} .
$$

Then (4.3) becomes

$$
\begin{aligned}
\left\|v_{\varepsilon}^{\sigma}\right\|_{H_{0}^{1}(\Omega)}^{2} & \leq c_{\Omega}^{2} c^{2}\left[\|f\|_{L^{2}(\Omega)}\left\|\bar{u}_{\varepsilon}^{\sigma}\right\|_{L^{2}\left(\Omega_{\varepsilon}\right)}+C\left\|\nabla \bar{u}_{\varepsilon}^{\sigma}\right\|_{L^{2}\left(\Omega_{\varepsilon}\right)}\right] \\
& \leq c_{\Omega}^{2} c^{2} \max \left\{\|f\|_{L^{2}}, C\right\}\left\|\bar{u}_{\varepsilon}^{\sigma}\right\|_{H^{1}\left(\Omega_{\varepsilon}\right)} \leq C_{\Omega}^{\prime}\left\|v_{\varepsilon}^{\sigma}\right\|_{H_{0}^{1}(\Omega)},
\end{aligned}
$$

which is

$$
\left\|v_{\varepsilon}^{\sigma}\right\|_{H_{0}^{1}(\Omega)} \leq C_{\Omega}^{\prime}
$$

where $C_{\Omega}^{\prime}$ is a constant independent of $\varepsilon$.

Now let us suppose

$$
0 \leq\left\|\bar{u}_{\varepsilon}^{\sigma}\right\|_{L^{4}\left(\Omega_{\varepsilon}\right)}<|\Omega|^{\frac{1}{4}} .
$$


By (4.2), inequality (4.3) becomes

$$
\left\|v_{\varepsilon}^{\sigma}\right\|_{H_{0}^{1}(\Omega)}^{2} \leq c_{\Omega}^{2} c^{2}\left[\|f\|_{L^{2}(\Omega)}\left\|\bar{u}_{\varepsilon}^{\sigma}\right\|_{L^{2}\left(\Omega_{\varepsilon}\right)}+|\Omega|+C\left\|\nabla \bar{u}_{\varepsilon}^{\sigma}\right\|_{L^{2}\left(\Omega_{\varepsilon}\right)}\right] .
$$

Then

$$
\left\|v_{\varepsilon}^{\sigma}\right\|_{H_{0}^{1}(\Omega)}^{2} \leq c_{\Omega}^{2} c^{2}\left[\max \left\{\|f\|_{L^{2}}, C\right\}\left\|\bar{u}_{\varepsilon}^{\sigma}\right\|_{H^{1}\left(\Omega_{\varepsilon}\right)}+|\Omega|\right],
$$

i.e. again

$$
\left\|v_{\varepsilon}^{\sigma}\right\|_{H_{0}^{1}(\Omega)} \leq C_{\Omega}^{\prime}
$$

where $C_{\Omega}^{\prime}$ is a constant independent of $\varepsilon$.

So there exists a subsequence, still denoted by $\varepsilon$, such that

$$
v_{\varepsilon}^{\sigma} \rightarrow u^{\sigma} \quad \text { weakly in } H_{0}^{1}(\Omega) .
$$

Obviously, since $v_{\varepsilon}^{\sigma}=u_{\varepsilon}^{\sigma}$ on $\Omega_{\varepsilon}^{\sigma}$, we deduce, by (4.6), that

$$
\left\|u_{\varepsilon}^{\sigma}\right\|_{H_{0}^{1}\left(\Omega_{\varepsilon}^{\sigma}\right)} \leq C_{\Omega}^{\prime}
$$

where $C_{\Omega}^{\prime}$ is a constant independent of $\varepsilon$.

By the Rellich theorem,

$$
\begin{aligned}
& \text { if } n=2, \quad v_{\varepsilon}^{\sigma} \in L^{q}, \text { for } q \in[1,+\infty[, \\
& \text { if } n \geq 3, \quad v_{\varepsilon}^{\sigma} \in L^{q}, \text { for } q \in\left[1, \frac{2 n}{n-2}\right],
\end{aligned}
$$

and

$$
v_{\varepsilon}^{\sigma} \rightarrow u^{\sigma} \quad \text { strongly in } L^{p}(\Omega), \begin{cases}\text { for } 1 \leq p<2^{*}=\frac{2 n}{n-2}, & \text { if } n>2, \\ \text { for } 2 \leq p<\infty, & \text { if } n=2,\end{cases}
$$

up to a subsequence still denoted by $\varepsilon$. Let us observe that

$$
\chi_{\Omega_{\varepsilon}^{\sigma}} \rightarrow \theta=\frac{\left|Y^{c}\right|}{|Y|} \quad \text { weakly* in } L^{\infty}(\Omega)
$$

Now we are able to prove the following result.

Lemma 4.1 Let $n=2$ and $n=3$. Let $\left(u_{\varepsilon}^{\sigma}\right)_{\varepsilon}$ be the sequence of solutions of problem (2.2), then

$$
\begin{aligned}
& \tilde{u}_{\varepsilon}^{\sigma} \rightarrow \theta u^{\sigma} \quad \text { weakly in } L^{2}(\Omega), \\
& \tilde{u}_{\varepsilon}^{\sigma}\left|\widetilde{u}_{\varepsilon}^{\sigma}\right|^{2} \rightarrow \theta u^{\sigma}\left|u^{\sigma}\right|^{2} \quad \text { weakly in } L^{\frac{4}{3}}(\Omega),
\end{aligned}
$$

up to a subsequence still denoted by $\varepsilon$. 
Proof By (4.11) we get

$$
\left\|\tilde{u}_{\varepsilon}^{\sigma}\right\|_{L^{2}(\Omega)}=\left\|u_{\varepsilon}^{\sigma}\right\|_{L^{2}\left(\Omega_{\varepsilon}^{\sigma}\right)} \leq C_{\Omega^{\prime}}^{\prime} .
$$

Then

$$
\tilde{u}_{\varepsilon}^{\sigma} \rightarrow z \quad \text { weakly in } L^{2}(\Omega),
$$

up to a subsequence still denoted by $\varepsilon$. Since

$$
\tilde{u}_{\varepsilon}^{\sigma}=\chi \Omega_{\varepsilon}^{\sigma} v_{\varepsilon}^{\sigma}
$$

by (4.13) and (4.14) we obtain

$$
z=\theta u^{\sigma}
$$

and then (4.15) holds. In order to prove (4.16) we observe that

$$
\left\|\tilde{u}_{\varepsilon}^{\sigma}\left|\widetilde{u}_{\varepsilon}^{\sigma}\right|^{2}\right\|_{L^{\frac{4}{3}(\Omega)}} \leq C
$$

where $C$ is a constant independent of $\varepsilon$. Indeed by (4.11) we have also

$$
\left\|\bar{u}_{\varepsilon}^{\sigma}\right\|_{H^{1}\left(\Omega_{\varepsilon}\right)} \leq C_{\Omega}^{\prime}
$$

where $C_{\Omega}^{\prime}$ is a constant independent of $\varepsilon$, which implies

$$
\left\|\nabla \bar{u}_{\varepsilon}^{\sigma}\right\|_{L^{2}\left(\Omega_{\varepsilon}\right)} \leq C_{\Omega}^{\prime}
$$

Multiplying by $u_{\varepsilon}^{\sigma}$ in (2.2) we get

$$
\int_{\Omega}\left|\widetilde{\nabla u_{\varepsilon}^{\sigma}}\right|^{2}+\int_{\Omega}\left|\widetilde{u}_{\varepsilon}^{\sigma}\right|^{4}=\int_{\Omega}\left|\widetilde{u}_{\varepsilon}^{\sigma}\right|^{2}+\int_{\Omega} f \widetilde{u}_{\varepsilon}^{\sigma}+\int_{\partial T_{\varepsilon}} g_{\varepsilon} \bar{u}_{\varepsilon}^{\sigma} .
$$

By (4.20) and (3.2) in Corollary 3.1 we get

$$
\int_{\Omega}\left|\widetilde{\nabla} u_{\varepsilon}^{\sigma}\right|^{2}+\int_{\Omega}\left|\widetilde{u}_{\varepsilon}^{\sigma}\right|^{4} \leq \int_{\Omega}\left|\widetilde{u}_{\varepsilon}^{\sigma}\right|^{2}+\int_{\Omega} f \widetilde{u}_{\varepsilon}^{\sigma}+C_{\Omega}^{\prime},
$$

which easily implies

$$
\int_{\Omega}\left|\tilde{u}_{\varepsilon}^{\sigma}\right|^{4} \leq C
$$

where $C$ is a constant independent of $\varepsilon$ and then (4.18) holds. As a consequence

$$
\widetilde{u}_{\varepsilon}^{\sigma}\left|\widetilde{u}_{\varepsilon}^{\sigma}\right|^{2} \rightarrow h \quad \text { weakly in } L^{\frac{4}{3}}(\Omega),
$$


up to a subsequence still denoted by $\varepsilon$. In order to identify $h$ we need to prove

$$
\left|v_{\varepsilon}^{\sigma}\right|^{2} \rightarrow\left|u^{\sigma}\right|^{2} \quad \text { strongly in } L^{2}(\Omega)
$$

To this aim we can write

$$
\begin{aligned}
\left\|\left|v_{\varepsilon}^{\sigma}\right|^{2}-\left|u^{\sigma}\right|^{2}\right\|_{L^{2}(\Omega)} & \leq 2 \int_{\Omega}\left|v_{\varepsilon}^{\sigma}-u^{\sigma}\right|^{2}\left(\left|v_{\varepsilon}^{\sigma}\right|^{2}+\left|u^{\sigma}\right|^{2}\right) d x \\
& \leq 2\left\|v_{\varepsilon}^{\sigma}-u^{\sigma}\right\|_{L^{4}(\Omega)}^{2}\left(\left\|v_{\varepsilon}^{\sigma}\right\|_{L^{4}(\Omega)}^{2}+\left\|u^{\sigma}\right\|_{L^{4}(\Omega)}^{2}\right) .
\end{aligned}
$$

By (4.25) and (4.13) we get (4.24) for $n=2$ and $n=3$.

Now, since

$$
\widetilde{u}_{\varepsilon}^{\sigma}\left|\tilde{u}_{\varepsilon}^{\sigma}\right|^{2}=\widetilde{u}_{\varepsilon}^{\sigma}\left|v_{\varepsilon}^{\sigma}\right|^{2}
$$

by (4.24) and (4.15) we get $h=\theta u^{\sigma}\left|u^{\sigma}\right|^{2}$ and then (4.16). So the lemma is completely proved.

Let us consider the function $w_{\lambda}^{\prime}$ defined as

$$
w_{\lambda}^{\prime}\left(x_{1}, x_{2}, \ldots, x_{n}\right)= \begin{cases}w_{\lambda}\left(x_{1}, x_{2}, \ldots, x_{n}\right), & \text { for } x \in Y^{c} \\ w_{\lambda}\left(-x_{1}, x_{2}, \ldots, x_{n}\right), & \text { for a.e. } x \in l Y \cap Q\end{cases}
$$

where $w_{\lambda}$ is the solution of problem (2.4).

Let us denote

$$
W_{\lambda}^{\prime}=\Phi w_{\lambda}^{\prime}\left(x_{1}, x_{2}, \ldots, x_{n}\right), \quad \text { for } x \in l Y \cap Q \text {, }
$$

where $\Phi$ is the extension operator defined by Lemma 3.1 with $C=Q \backslash l Y$ and $R=Y$. From problem (2.4) we can note that

$$
W_{\lambda}^{\prime}(y)=\lambda y+v_{\lambda}(y), \quad \text { for } y \in Y \text {, }
$$

where $v_{\lambda}$ is an $Y$-periodic function. Let us denote by

$$
\mathcal{P} W_{\lambda}^{\prime}(y+\mathbf{k})=\lambda(y+\mathbf{k})+v_{\lambda}(y+\mathbf{k})=\lambda y+v_{\lambda}(y)+\lambda \mathbf{k}=W_{\lambda}^{\prime}(y)+\lambda \mathbf{k}
$$

for $y \in Y$ and for $\mathbf{k} \in \mathbf{Z}^{n}$ the periodic extension of $W_{\lambda}^{\prime}(y)$ on $\mathbf{R}^{n}$. Finally let us set

$$
w_{\varepsilon, \lambda}=\varepsilon\left(\mathcal{P} W_{\lambda}^{\prime}\right)\left(\frac{x}{\varepsilon}\right), \quad \text { for } x \in \mathbf{R}^{n} .
$$

It can be proved that (see (4.6)-(4.19) of [5])

$$
\left\|w_{\varepsilon, \lambda}\right\|_{L^{\infty}(\Omega)} \leq c
$$

where $c$ is a constant independent of $\varepsilon$, and

$$
w_{\varepsilon, \lambda}(x) \rightarrow \lambda x=w_{\lambda}^{*} \quad \text { strongly in } L^{\infty}(\Omega),
$$




$$
w_{\varepsilon, \lambda}(x) \rightarrow \lambda x=w_{\lambda}^{*} \quad \text { weakly in } W^{1, p}(\Omega)
$$

for $p$ such that

$$
\begin{array}{ll}
2<p \leq \frac{4}{2-2 \alpha}, & \text { if } n=2, \\
3<p \leq \frac{6}{3-2 \alpha}, & \text { if } n=3, \\
1 \leq p<+\infty, & \text { if } n>3,
\end{array}
$$

with $\left.\alpha \in] \frac{1}{2}, 1\right]$.

It is easy to see also that (see (4.19) of [5])

$$
\begin{aligned}
& \left\|w_{\varepsilon, \lambda}\right\|_{W^{1, p}(\Omega)} \leq C, \quad 2<p \leq \frac{4}{2-2 \alpha}\left(\frac{4}{2-2 \alpha}>4\right), \text { if } n=2, \\
& \left\|w_{\varepsilon, \lambda}\right\|_{W^{1, p}(\Omega)} \leq C, \quad 3<p \leq \frac{6}{3-2 \alpha}\left(\frac{6}{3-2 \alpha}>3\right), \text { if } n=3, \\
& \left\|w_{\varepsilon, \lambda}\right\|_{W^{1, p}(\Omega)} \leq C, \quad 1 \leq p<+\infty, \text { if } n>3 .
\end{aligned}
$$

Let us pose $\eta_{\varepsilon, \lambda}=\nabla \mathcal{P} W_{\lambda}^{\prime}\left(\frac{x}{\varepsilon}\right)$ in $\Omega_{\varepsilon}$. This function satisfies the problem

$$
\begin{cases}-\operatorname{div} \eta_{\varepsilon, \lambda}=0, & \text { in } \Omega_{\varepsilon} \\ \eta_{\varepsilon, \lambda} \cdot \mathbf{n}=0, & \text { on } \partial T_{\varepsilon}\end{cases}
$$

whose variational formulation, by the periodicity of $w_{\varepsilon, \lambda}$, is

$$
\int_{\Omega} \tilde{\eta}_{\varepsilon, \lambda} \nabla \varphi=0, \quad \forall \varphi \in H^{1}(\Omega)
$$

Moreover, we have

$$
\tilde{\eta}_{\varepsilon, \lambda}(x) \rightarrow \mathcal{A} \lambda \quad \text { weakly* in }\left(L^{2}(\Omega)\right)^{n}
$$

\subsection{Identification of the limit problem}

Let us pose $\xi_{\varepsilon}^{\sigma}=\left(\xi_{\varepsilon, 1}^{\sigma}, \xi_{\varepsilon, 2}^{\sigma}\right)$ where $\xi_{\varepsilon, i}^{\sigma}=\nabla u_{\varepsilon, i}^{\sigma}$ for $i=1,2$ and observe that by (2.2) we get

$$
\begin{cases}-\operatorname{div} \xi_{\varepsilon, i}^{\sigma}-u_{\varepsilon, i}^{\sigma}+\left|u_{\varepsilon}^{\sigma}\right|^{2} u_{\varepsilon, i}^{\sigma}=f_{i}, & \text { in } \Omega_{\varepsilon}^{\sigma}, \\ u_{\varepsilon}^{\sigma}=0, & \text { on } \partial \Omega \cup \Gamma_{\varepsilon}^{D, \sigma}, \\ \xi_{\varepsilon, i}^{\sigma} \cdot \mathbf{n}=g_{\varepsilon, i}, & \text { on } \Gamma_{\varepsilon}^{N, \sigma},\end{cases}
$$

for $i=1,2$. Moreover, by (4.11) we obtain

$$
\widetilde{\xi}_{\varepsilon, i}^{\sigma} \rightarrow \xi_{*, i}^{\sigma} \quad \text { weakly in }\left(L^{2}(\Omega)\right)^{n}
$$

for $i=1,2$.

Now, let us fix $i$ and observe that problem (4.35) implies

$$
\int_{\Omega}\left\langle\widetilde{\xi}_{\varepsilon, i}^{\sigma}, \nabla \varphi\right\rangle-\int_{\Gamma_{\varepsilon}^{N, \sigma}} g_{\varepsilon, i} \varphi d \sigma=\int_{\Omega} \widetilde{u}_{\varepsilon, i}^{\sigma} \varphi-\int_{\Omega}\left|\widetilde{u}_{\varepsilon, i}^{\sigma}\right|^{2} \widetilde{u}_{\varepsilon}^{\sigma} \varphi+\int_{\Omega} \chi_{\Omega_{\varepsilon}^{\sigma}} f_{i} \varphi, \quad \forall \varphi \in V_{\varepsilon}^{\sigma} .
$$


Let us determine $\xi_{*, i}^{\sigma}$. For $i=1,2$ let $\vartheta_{g_{i}} \in H^{1}\left(Y^{c}\right)$ be the function such that

$$
\begin{cases}-\Delta \vartheta_{g_{i}}=0, & \text { in } Y^{c}, \\ \frac{\partial \vartheta_{g_{i}}}{\partial \mathbf{n}}=g_{i}, & \text { on } \partial Q, \\ \vartheta_{g_{i}}, & Y \text {-periodic s.t. } m_{Y^{c}}\left(\vartheta_{g_{i}}\right)=0,\end{cases}
$$

and let us pose

$$
\gamma_{i}=\nabla_{y} \vartheta_{g_{i}}, \quad \text { in } Y^{c}
$$

Let $\gamma_{\varepsilon, i}$ be the function defined as

$$
\gamma_{\varepsilon, i}(x)=\widehat{\gamma}_{i}\left(\frac{x}{\varepsilon}\right), \quad \text { in } \mathbf{R}^{n} \backslash \bigcup_{k \in \mathbf{Z}^{n}} \varepsilon(Q+k)
$$

for $i=1,2$. Using (4.38), $\gamma_{\varepsilon, i}$ verifies the following problem:

$$
\begin{cases}-\operatorname{div} \gamma_{\varepsilon, i}=0, & \text { in } \bigcup_{k} \varepsilon\left(Y^{c}+k\right), \\ \gamma_{\varepsilon, i} \cdot \mathbf{n}=g_{\varepsilon, i}, & \text { on } \bigcup_{k} \partial\left(\varepsilon\left(Y^{c}+k\right)\right) .\end{cases}
$$

Moreover, by the periodicity of $\gamma_{\varepsilon, i}$, we have

$$
\tilde{\gamma}_{\varepsilon, i} \rightarrow m_{Y^{c}}\left(\gamma_{i}\right) \quad \text { weakly in }\left(L^{2}(\Omega)\right)^{n} \text {. }
$$

Let us pose $\vartheta_{g_{i}}^{\varepsilon}=\varepsilon \widehat{\vartheta}_{i}\left(\frac{x}{\varepsilon}\right)$. The function $P_{\varepsilon} \vartheta_{g_{i}}^{\varepsilon}$, where $\left\{P_{\varepsilon}\right\}_{\varepsilon}$ is the sequence of extension operators given by Theorem 3.1, is bounded in $H^{1}(\Omega)$.

In particular, $\vartheta_{g_{i}}^{\varepsilon}$ is a null average function, so we obtain

$$
\widetilde{\vartheta}_{g_{i}}^{\varepsilon} \rightarrow 0 \text { strongly in } L^{2}(\Omega)
$$

Instead of problem (4.37) we can consider the following one:

$$
\begin{aligned}
\int_{\Omega}\left(\widetilde{\xi}_{\varepsilon, i}^{\sigma}-\widetilde{\gamma}_{\varepsilon, i}\right) \nabla \varphi & =\int_{\Omega_{\varepsilon}^{\sigma}}\left(\widetilde{\xi}_{\varepsilon, i}^{\sigma}-\gamma_{\varepsilon, i}\right) \nabla \varphi \\
& =\int_{\Omega} \widetilde{u}_{\varepsilon, i}^{\sigma} \varphi-\int_{\Omega}\left|\widetilde{u}_{\varepsilon}^{\sigma}\right|^{2} \widetilde{u}_{\varepsilon, i}^{\sigma} \varphi+\int_{\Omega} \chi_{\Omega_{\varepsilon}^{\sigma}} f_{i} \varphi, \quad \forall \varphi \in V_{\varepsilon}^{\sigma} .
\end{aligned}
$$

If we take $\varphi w_{\varepsilon, \lambda} \psi_{\varepsilon}^{\sigma}$ as a test function in (4.43), where $\psi_{\varepsilon}^{\sigma}$ is given in Lemma 3.2, and $\varphi \in$ $\mathcal{D}(\Omega)$, using the extension to zero on the whole $\Omega$, we obtain

$$
\begin{gathered}
\int_{\Omega}\left(\widetilde{\xi}_{\varepsilon, i}^{\sigma}-\tilde{\gamma}_{\varepsilon, i}\right) \nabla \varphi\left(w_{\varepsilon, \lambda} \psi_{\varepsilon}^{\sigma}\right)+\int_{\Omega}\left(\widetilde{\xi}_{\varepsilon, i}^{\sigma}-\widetilde{\gamma}_{\varepsilon, i}\right) \widetilde{\eta}_{\varepsilon, \lambda}\left(\varphi \psi_{\varepsilon}^{\sigma}\right)+\int_{\Omega}\left(\widetilde{\xi}_{\varepsilon, i}^{\sigma}-\widetilde{\gamma}_{\varepsilon, i}\right) \nabla \psi_{\varepsilon}^{\sigma}\left(w_{\varepsilon, \lambda} \varphi\right) \\
=\int_{\Omega} \widetilde{u}_{\varepsilon, i}^{\sigma}\left(\varphi w_{\varepsilon, \lambda} \psi_{\varepsilon}^{\sigma}\right)-\int_{\Omega}\left|\widetilde{u}_{\varepsilon}^{\sigma}\right|^{2} \widetilde{u}_{\varepsilon, i}^{\sigma}\left(\varphi w_{\varepsilon, \lambda} \psi_{\varepsilon}^{\sigma}\right)+\int_{\Omega} \chi_{\Omega_{\varepsilon}^{\sigma}} f_{i}\left(\varphi w_{\varepsilon, \lambda} \psi_{\varepsilon}^{\sigma}\right) .
\end{gathered}
$$

Moreover, if we take $\varphi\left(v_{\varepsilon, i}^{\sigma}-\widetilde{\vartheta}_{g_{i}}^{\varepsilon}\right)$ as test function in problem (4.33), we have

$$
\int_{\Omega} \tilde{\eta}_{\varepsilon, \lambda} \nabla \varphi\left(v_{\varepsilon, i}^{\sigma}-\tilde{\vartheta}_{g_{i}}^{\varepsilon}\right)+\int_{\Omega} \tilde{\eta}_{\varepsilon, \lambda} \varphi \nabla\left(v_{\varepsilon, i}^{\sigma}-\widetilde{\vartheta}_{g_{i}}^{\varepsilon}\right)=0
$$


By (4.44) and (4.45), we obtain

$$
\begin{aligned}
& \int_{\Omega}\left(\widetilde{\xi}_{\varepsilon, i}^{\sigma}-\tilde{\gamma}_{\varepsilon, i}\right) \nabla \varphi\left(w_{\varepsilon, \lambda} \psi_{\varepsilon}^{\sigma}\right)+\int_{\Omega}\left(\tilde{\xi}_{\varepsilon, i}^{\sigma}-\tilde{\gamma}_{\varepsilon, i}\right) \tilde{\eta}_{\varepsilon, \lambda}\left(\varphi \psi_{\varepsilon}^{\sigma}\right)-\int_{\Omega} \tilde{\eta}_{\varepsilon, \lambda} \varphi \nabla\left(v_{\varepsilon, i}^{\sigma}-\tilde{\vartheta}_{g_{i}}^{\varepsilon}\right) \\
& \quad+\int_{\Omega}\left(\widetilde{\xi}_{\varepsilon, i}^{\sigma}-\widetilde{\gamma}_{\varepsilon, i}\right) \nabla \psi_{\varepsilon}^{\sigma}\left(w_{\varepsilon, \lambda} \varphi\right)-\int_{\Omega} \tilde{\eta}_{\varepsilon, \lambda} \nabla \varphi\left(v_{\varepsilon, i}^{\sigma}-\widetilde{\vartheta}_{g_{i}}^{\varepsilon}\right) \\
& =\int_{\Omega} \tilde{u}_{\varepsilon, i}\left(\varphi w_{\varepsilon, \lambda} \psi_{\varepsilon}^{\sigma}\right)-\int_{\Omega}\left|\tilde{u}_{\varepsilon}^{\sigma}\right|^{2} \tilde{u}_{\varepsilon, i}^{\sigma}\left(\varphi w_{\varepsilon, \lambda} \psi_{\varepsilon}^{\sigma}\right)+\int_{\Omega} \chi_{\Omega_{\varepsilon}^{\sigma}} f_{i}\left(\varphi w_{\varepsilon, \lambda} \psi_{\varepsilon}^{\sigma}\right) .
\end{aligned}
$$

Since $0 \leq \psi_{\varepsilon}^{\sigma} \leq 1$, and the support of $\left(\psi_{\varepsilon}^{\sigma}-1\right)$ is included in $A_{\varepsilon}$, the second and the third term in our formulation can be majorized.

In fact

$$
\begin{aligned}
& \int_{\Omega}\left(\widetilde{\xi}_{\varepsilon, i}^{\sigma}-\tilde{\gamma}_{\varepsilon, i}\right) \tilde{\eta}_{\varepsilon, \lambda}\left(\varphi \psi_{\varepsilon}^{\sigma}\right)-\int_{\Omega} \tilde{\eta}_{\varepsilon, \lambda} \varphi \nabla\left(v_{\varepsilon, i}^{\sigma}-\widetilde{\vartheta}_{g_{i}}^{\varepsilon}\right) \\
& =\int_{\Omega}\left(\widetilde{\xi}_{\varepsilon, i}^{\sigma}-\tilde{\gamma}_{\varepsilon, i}\right) \tilde{\eta}_{\varepsilon, \lambda}\left(\varphi \psi_{\varepsilon}^{\sigma}\right)-\int_{\Omega} \tilde{\eta}_{\varepsilon, \lambda} \varphi\left(\widetilde{\xi}_{\varepsilon, i}^{\sigma}-\widetilde{\gamma}_{\varepsilon, i}\right) \\
& \leq\left|\int_{\Omega}\left(\widetilde{\xi}_{\varepsilon, i}^{\sigma}-\tilde{\gamma}_{\varepsilon, i}\right) \tilde{\eta}_{\varepsilon, \lambda} \varphi\left(\psi_{\varepsilon}^{\sigma}-1\right)\right| \\
& =\left|\int_{\Omega_{\varepsilon}^{\sigma}}\left(\widetilde{\xi}_{\varepsilon, i}^{\sigma}-\tilde{\gamma}_{\varepsilon, i}\right) \tilde{\eta}_{\varepsilon, \lambda} \varphi\left(\psi_{\varepsilon}^{\sigma}-1\right)\right| \\
& \leq\|\varphi\|_{L^{\infty}(\Omega)}\left\|\left(\xi_{\varepsilon, i}^{\sigma}-\widetilde{\gamma}_{\varepsilon, i}\right)\right\|_{\left(L^{2}\left(\Omega_{\varepsilon}^{\sigma}\right)\right)^{n}}\left\|\eta_{\varepsilon, \lambda}\right\|_{\left(L^{2}\left(A_{\varepsilon} \cap \Omega_{\varepsilon}^{\sigma}\right)\right)^{n}} \\
& \leq C_{1} \varepsilon^{n}\left\|\nabla \Phi w_{\lambda}^{\prime}\right\|_{\left(L^{2}\left(\frac{A_{\varepsilon}}{\varepsilon}\right)\right)^{n}} \leq C_{2}|\Omega|\left\|\nabla \Phi w_{\lambda}^{\prime}\right\|_{\left(L^{2}\left(\frac{A_{\varepsilon}}{\varepsilon} \cap Y\right)\right)^{n}} .
\end{aligned}
$$

By the absolute continuity of the integral and as $\left|\frac{A_{\varepsilon}}{\varepsilon}\right| \rightarrow 0$, we get $\left\|\nabla \Phi w_{\lambda}^{\prime}\right\|_{\left(L^{2}\left(\frac{A_{\varepsilon}}{\varepsilon} \cap Y\right)\right)^{n}}$ tending to zero as $\varepsilon \rightarrow 0$.

By (3.10) and by (4.9) we obtain the following convergence result:

$$
w_{\varepsilon, \lambda} \psi_{\varepsilon}^{\sigma} \rightarrow w_{\lambda}^{*} \quad \text { strongly in } L^{2}(\Omega)
$$

Then by (4.36), (4.41), and (4.47), we have, as $\varepsilon \rightarrow 0$,

$$
\int_{\Omega}\left(\widetilde{\xi}_{\varepsilon, i}^{\sigma}-\widetilde{\gamma}_{\varepsilon, i}\right) \nabla \varphi\left(w_{\varepsilon, \lambda} \psi_{\varepsilon}^{\sigma}\right) \longrightarrow \int_{\Omega}\left(\xi_{*, i}^{\sigma}-m_{Y^{c}}\left(\gamma_{i}\right)\right) \nabla \varphi w_{\lambda}^{*}
$$

By (4.13), (4.34), and (4.42) we get

$$
\int_{\Omega} \tilde{\eta}_{\varepsilon, \lambda} \nabla \varphi\left(v_{\varepsilon, i}^{\sigma}-\widetilde{\vartheta}_{g_{i}}^{\varepsilon}\right) \longrightarrow \int_{\Omega} \mathcal{A} \lambda \nabla \varphi u_{i}^{\sigma}
$$

Moreover, for $n=2$ and $n=3$, by (4.10), (4.14), (4.15), and (4.47) we have

$$
\begin{gathered}
\int_{\Omega} \chi_{\Omega_{\varepsilon}^{\sigma}} f_{i}\left(\varphi w_{\varepsilon, \lambda} \psi_{\varepsilon}^{\sigma}\right) \rightarrow \int_{\Omega} \theta f_{i} \varphi w_{\lambda}^{*}, \\
\int_{\Omega} \tilde{u}_{\varepsilon, i}^{\sigma}\left(\varphi w_{\varepsilon, \lambda} \psi_{\varepsilon}^{\sigma}\right) \longrightarrow \int_{\Omega} \theta u_{i}^{\sigma} \varphi w_{\lambda}^{*} .
\end{gathered}
$$


By (3.10), (4.16), and (4.29) we get

$$
\int_{\Omega}\left|\widetilde{u}_{\varepsilon}^{\sigma}\right|^{2} \widetilde{u}_{\varepsilon, i}^{\sigma}\left(\varphi w_{\varepsilon, \lambda} \psi_{\varepsilon}^{\sigma}\right) \rightarrow \int_{\Omega} \theta\left|u^{\sigma}\right|^{2} u_{i}^{\sigma} \varphi w_{\lambda}^{*}
$$

for $n=2$ and $n=3$.

Let us consider the fourth term in (4.46).

Let $\left(\phi_{\varepsilon}\right)_{\varepsilon}$ be a sequence in $L^{\infty}(\Omega)$ such that

$$
\phi_{\varepsilon} \longrightarrow \varphi \text { strongly in } L^{\infty}(\Omega)
$$

and $\phi_{\varepsilon}$ is constant on $\varepsilon^{v} B+\varepsilon k$, for every $\varepsilon, k \in \mathbf{Z}^{n}$ such that $\varepsilon(Y+k) \subset \Omega$.

Since $\psi_{\varepsilon}^{\sigma}=1$ in $\Omega \backslash A_{\varepsilon}$ and $\nabla \psi_{\varepsilon}^{\sigma}=0$ in $\Omega \backslash A_{\varepsilon}$, we have

$$
\begin{aligned}
\int_{\Omega} & \left(\widetilde{\xi}_{\varepsilon, i}^{\sigma}-\widetilde{\gamma}_{\varepsilon, i}\right) \nabla \psi_{\varepsilon}^{\sigma}\left(w_{\varepsilon, \lambda} \varphi\right) \\
= & \int_{\Omega_{\varepsilon}}\left(\widetilde{\xi}_{\varepsilon, i}^{\sigma}-\widetilde{\gamma}_{\varepsilon, i}\right) \nabla \psi_{\varepsilon}^{\sigma}\left(w_{\varepsilon, \lambda} \varphi\right) \\
= & \left.\int_{\Omega_{\varepsilon}} \varphi w_{\varepsilon, \lambda} \widetilde{\xi}_{\varepsilon, i}^{\sigma}-\gamma_{\varepsilon, i}\right) \nabla \psi_{\varepsilon}^{\sigma}-\int_{\Omega_{\varepsilon} \cap A_{\varepsilon}} \phi_{\varepsilon} w_{\varepsilon, \lambda}\left(\widetilde{\xi}_{\varepsilon, i}^{\sigma}-\widetilde{\gamma}_{\varepsilon, i}\right) w_{\varepsilon, \lambda} \nabla \psi_{\varepsilon}^{\sigma} \\
& +\int_{\Omega_{\varepsilon} \cap A_{\varepsilon}}\left(\widetilde{\xi}_{\varepsilon, i}^{\sigma}-\widetilde{\gamma}_{\varepsilon, i}\right) w_{\varepsilon, \lambda} \nabla \psi_{\varepsilon}^{\sigma} \phi_{\varepsilon} \\
= & \int_{\Omega_{\varepsilon} \cap A_{\varepsilon}}\left(\widetilde{\xi}_{\varepsilon, i}^{\sigma}-\widetilde{\gamma}_{\varepsilon, i}\right) w_{\varepsilon, \lambda} \nabla \psi_{\varepsilon}^{\sigma}\left(\varphi-\phi_{\varepsilon}\right)+\int_{\Omega_{\varepsilon} \cap A_{\varepsilon}} \phi_{\varepsilon}\left(\widetilde{\xi}_{\varepsilon, i}^{\sigma}-\widetilde{\gamma}_{\varepsilon, i}\right) w_{\varepsilon, \lambda} \nabla \psi_{\varepsilon}^{\sigma} .
\end{aligned}
$$

Denoting by $P_{\varepsilon}$ the extension operators given by Theorem 3.6, by the properties of the symmetry of $\psi_{\varepsilon}^{\sigma}$ given by (3.12), $w_{\varepsilon, \lambda}$, and since $\phi_{\varepsilon}$ is constant on $\Omega_{\varepsilon} \cap A_{\varepsilon}$, we have

$$
\begin{aligned}
\int_{\Omega_{\varepsilon} \cap A_{\varepsilon}} \phi_{\varepsilon} w_{\varepsilon, \lambda}\left(\tilde{\xi}_{\varepsilon, i}^{\sigma}-\tilde{\gamma}_{\varepsilon, i}\right) \nabla \psi_{\varepsilon}^{\sigma} & =\int_{\Omega_{\varepsilon} \cap A_{\varepsilon}} \phi_{\varepsilon} w_{\varepsilon, \lambda}\left(\nabla u_{\varepsilon, i}^{\sigma}-\nabla P_{\varepsilon} \vartheta_{g_{i}}^{\varepsilon}\right) \nabla \psi_{\varepsilon}^{\sigma} \\
& =\frac{1}{2} \int_{A_{\varepsilon}} \phi_{\varepsilon} w_{\varepsilon, \lambda}\left(\nabla P_{\varepsilon} u_{\varepsilon, i}^{\sigma}-\nabla P_{\varepsilon} \vartheta_{g_{i}}^{\varepsilon}\right) \nabla \psi_{\varepsilon}^{\sigma} .
\end{aligned}
$$

By (4.54) and (4.55) we obtain

$$
\begin{aligned}
\int_{\Omega}\left(\widetilde{\xi}_{\varepsilon, i}^{\sigma}-\widetilde{\gamma}_{\varepsilon, i}\right) \nabla \psi_{\varepsilon}^{\sigma}\left(w_{\varepsilon, \lambda} \varphi\right) \\
=\int_{\Omega_{\varepsilon}}\left(\widetilde{\xi}_{\varepsilon, i}^{\sigma}-\gamma_{\varepsilon, i}\right) w_{\varepsilon, \lambda} \nabla \psi_{\varepsilon}^{\sigma}\left(\varphi-\phi_{\varepsilon}\right) \\
\quad+\frac{1}{2} \int_{A_{\varepsilon}} \phi_{\varepsilon} w_{\varepsilon, \lambda}\left(\nabla P_{\varepsilon} u_{\varepsilon, i}^{\sigma}-\nabla P_{\varepsilon} \vartheta_{g_{i}}^{\varepsilon}\right) \nabla \psi_{\varepsilon}^{\sigma} \\
\quad-\frac{1}{2} \int_{A_{\varepsilon}} \varphi w_{\varepsilon, \lambda}\left(\nabla P_{\varepsilon} u_{\varepsilon, i}^{\sigma}-\nabla P_{\varepsilon} \vartheta_{g_{i}}^{\varepsilon}\right) \nabla \psi_{\varepsilon}^{\sigma}+\frac{1}{2} \int_{A_{\varepsilon}} \varphi w_{\varepsilon, \lambda}\left(\nabla P_{\varepsilon} u_{\varepsilon, i}^{\sigma}-\nabla P_{\varepsilon} \vartheta_{g_{i}}^{\varepsilon}\right) \nabla \psi_{\varepsilon}^{\sigma} \\
=\int_{\Omega_{\varepsilon}}\left(\widetilde{\xi}_{\varepsilon, i}^{\sigma}-\gamma_{\varepsilon, i}\right) w_{\varepsilon, \lambda} \nabla \psi_{\varepsilon}^{\sigma}\left(\varphi-\phi_{\varepsilon}\right)+\frac{1}{2} \int_{A_{\varepsilon}}\left(\phi_{\varepsilon}-\varphi\right)\left(\nabla P_{\varepsilon} u_{\varepsilon, i}^{\sigma}-\nabla P_{\varepsilon} \vartheta_{g_{i}}^{\varepsilon}\right) w_{\varepsilon, \lambda} \nabla \psi_{\varepsilon}^{\sigma} \\
\quad+\frac{1}{2} \int_{A_{\varepsilon}} \varphi\left(\nabla P_{\varepsilon} u_{\varepsilon, i}^{\sigma}-\nabla P_{\varepsilon} \vartheta_{g_{i}}^{\varepsilon}\right) w_{\varepsilon, \lambda} \nabla \psi_{\varepsilon}^{\sigma} .
\end{aligned}
$$


By (3.9), (4.27), (4.36), (4.41), (4.53) we obtain

$$
\begin{aligned}
& \left|\int_{\Omega_{\varepsilon}}\left(\widetilde{\xi}_{\varepsilon, i}^{\sigma}-\gamma_{\varepsilon, i}\right) w_{\varepsilon, \lambda} \nabla \psi_{\varepsilon}^{\sigma}\left(\varphi-\phi_{\varepsilon}\right)\right| \\
& \quad \leq\left\|\varphi-\phi_{\varepsilon}\right\|_{L^{\infty}(\Omega)}\left\|w_{\varepsilon, \lambda}\right\|_{L^{\infty}(\Omega)}\left\|\left(\widetilde{\xi}_{\varepsilon, i}^{\sigma}-\widetilde{\gamma}_{\varepsilon, i}\right)\right\|_{\left(L^{2}(\Omega)\right)^{n}}\left\|\nabla \psi_{\varepsilon}^{\sigma}\right\|_{L^{2}(\Omega)} .
\end{aligned}
$$

The last expression tends to zero as the first term of the product tends to zero and the other ones are bounded. In a similar way we obtain

$$
\frac{1}{2} \int_{A_{\varepsilon}}\left(\varphi-\phi_{\varepsilon}\right)\left(\nabla P_{\varepsilon} u_{\varepsilon, i}^{\sigma}-\nabla P_{\varepsilon} \vartheta_{g_{i}}^{\varepsilon}\right) w_{\varepsilon, \lambda} \nabla \psi_{\varepsilon}^{\sigma} \longrightarrow 0
$$

Let us consider the last term in (4.56) and observe that

$$
\begin{aligned}
& \frac{1}{2} \int_{A_{\varepsilon}} \varphi\left(\nabla P_{\varepsilon} u_{\varepsilon, i}^{\sigma}-\nabla P_{\varepsilon} \vartheta_{g_{i}}^{\varepsilon}\right) w_{\varepsilon, \lambda} \nabla \psi_{\varepsilon}^{\sigma} \\
& \quad=\frac{1}{2} \int_{\Omega} \varphi \nabla P_{\varepsilon} u_{\varepsilon, i}^{\sigma} w_{\varepsilon, \lambda} \nabla \psi_{\varepsilon}^{\sigma}-\frac{1}{2} \int_{A_{\varepsilon}} \varphi \nabla P_{\varepsilon} \vartheta_{g_{i}}^{\varepsilon} w_{\varepsilon, \lambda} \nabla \psi_{\varepsilon}^{\sigma} \\
& \quad=\frac{1}{2} \int_{\Omega} \varphi \nabla v_{\varepsilon, i}^{\sigma} w_{\varepsilon, \lambda} \nabla \psi_{\varepsilon}^{\sigma}-\frac{1}{2} \int_{A_{\varepsilon}} \varphi \nabla P_{\varepsilon} \vartheta_{g_{i}}^{\varepsilon} w_{\varepsilon, \lambda} \nabla \psi_{\varepsilon}^{\sigma} .
\end{aligned}
$$

Let us analyze these two terms separately. We observe that

$$
\int_{\Omega} \varphi \nabla v_{\varepsilon, i}^{\sigma} w_{\varepsilon, \lambda} \nabla \psi_{\varepsilon}^{\sigma}=\int_{A_{\varepsilon}} \varphi \nabla v_{\varepsilon, i}^{\sigma} w_{\varepsilon, \lambda} \nabla \psi_{\varepsilon}^{\sigma}
$$

and

$$
w_{\varepsilon, \lambda} v_{\varepsilon, i}^{\sigma} \varphi \in H_{0}^{1}\left(\Omega \backslash D_{\varepsilon}^{\prime \sigma}\right) .
$$

In fact since $v_{\varepsilon, i}^{\sigma}=0$ on $D_{\varepsilon}^{\prime \sigma}$ and $\varphi \in \mathcal{D}(\Omega)$, by Corollary 3.1 we must only prove that $w_{\varepsilon, \lambda} v_{\varepsilon, i}^{\sigma} \in H^{1}(\Omega)$. Obviously $\nabla\left(w_{\varepsilon, \lambda} v_{\varepsilon, i}^{\sigma}\right)=w_{\varepsilon, \lambda} \nabla v_{\varepsilon, i}^{\sigma}+v_{\varepsilon, i}^{\sigma} \nabla w_{\varepsilon, \lambda}$. By (4.28) and since $\nabla v_{\varepsilon, i}^{\sigma} \in$ $L^{2}(\Omega)$ we have $w_{\varepsilon, \lambda} \nabla v_{\varepsilon, i}^{\sigma} \in L^{2}(\Omega)$ If $n \leq 3$, by (4.12) and (4.32) it follows that $\nabla w_{\varepsilon, \lambda} v_{\varepsilon, i}^{\sigma} \in$ $L^{r}(\Omega) \forall r<p$. So we have

$$
\begin{aligned}
& \int_{\Omega} \varphi \nabla v_{\varepsilon, i}^{\sigma} w_{\varepsilon, \lambda} \nabla \psi_{\varepsilon}^{\sigma} \\
& \quad=\left\langle-\Delta \psi_{\varepsilon}^{\sigma}, v_{\varepsilon, i}^{\sigma} w_{\varepsilon, \lambda} \varphi\right\rangle-\int_{\Omega} \nabla \varphi \nabla \psi_{\varepsilon}^{\tau}\left(v_{\varepsilon, i}^{\sigma} w_{\varepsilon, \lambda}\right)-\int_{\Omega} \nabla \psi_{\varepsilon}^{\sigma} \nabla w_{\varepsilon, \lambda} v_{\varepsilon, i}^{\sigma} \varphi .
\end{aligned}
$$

By the equiboundedness of $w_{\varepsilon}^{\sigma}$ in $L^{\infty}(\Omega)$, by (4.13) and since, by (3.9), $\nabla \psi_{\varepsilon}^{\sigma} \rightarrow 0$ weakly in $L^{2}(\Omega)$, we see that $\int_{\Omega} \nabla \varphi \nabla \psi_{\varepsilon}^{\sigma}\left(v_{\varepsilon, i}^{\sigma} w_{\varepsilon, \lambda}\right)$ tends to zero. We can observe that

$$
v_{\varepsilon, i}^{\sigma} \nabla w_{\varepsilon, \lambda} \text { is equibounded in } L^{s}(\Omega) \text { with } s>2 .
$$

In fact if $n=2$, by (4.32) we have the result that $\nabla w_{\varepsilon, \lambda}$ is equibounded in $L^{r}(\Omega)$ with $r>2$. By (4.12) $v_{\varepsilon, i}^{\sigma}$ is equibounded in $L^{q}(\Omega)$ for $1 \leq q<+\infty$ and so by (4.63) there exists $s>2$ such that $v_{\varepsilon}^{\sigma} \nabla w_{\varepsilon, \lambda}$ is equibounded in $L^{s}(\Omega)$. By (3.11), $\nabla \psi_{\varepsilon}^{\sigma} \rightarrow 0$ strongly in $L^{s^{\prime}}(\Omega)$, with $\frac{1}{s}+\frac{1}{s^{\prime}}=1$. 
If $n=3$, by (4.32) there exists $r$ such that $\left.\left.3<r \leq \frac{6}{3-2 \alpha}(\alpha \in] \frac{1}{2}, 1\right]\right)$ and $\nabla w_{\varepsilon, \lambda}$ is equibounded in $L^{r}(\Omega)$. By (4.12) $v_{\varepsilon, i}^{\sigma}$ is equibounded in $L^{q}(\Omega)$ for $1 \leq q<6$. So there exists $s>2$ such that $v_{\varepsilon, i}^{\sigma} \nabla w_{\varepsilon, \lambda}$ is equibounded in $L^{s}(\Omega)$. Then by (3.11) $\nabla \psi_{\varepsilon}^{\sigma} \longrightarrow 0$ strongly in $L^{s^{\prime}}(\Omega)$, with $\frac{1}{s}+\frac{1}{s^{\prime}}=1$.

So we find that $\int_{\Omega} \nabla \psi_{\varepsilon}^{\sigma} \nabla w_{\varepsilon, \lambda} v_{\varepsilon, i}^{\sigma} \varphi$ tends to zero.

As by (4.10) $v_{\varepsilon, i}^{\sigma} \longrightarrow u_{i}^{\sigma}$ strongly in $L^{2}(\Omega)$ and as by (4.29) $w_{\varepsilon, \lambda} \longrightarrow w_{\lambda}^{*}$ strongly in $L^{\infty}(\Omega)$, we have

$$
\begin{aligned}
\left\|v_{\varepsilon, i}^{\sigma} w_{\varepsilon, \lambda}-u_{i}^{\sigma} \lambda x\right\|_{L^{2}(\Omega)} & =\left\|v_{\varepsilon, i}^{\sigma} w_{\varepsilon, \lambda}-u_{i}^{\sigma} w_{\varepsilon, \lambda}+u_{i}^{\sigma} w_{\varepsilon, \lambda}-u_{i}^{\sigma} \lambda x\right\|_{L^{2}(\Omega)} \\
& \leq\left\|v_{\varepsilon, i}^{\sigma}-u_{i}^{\sigma}\right\|_{L^{2}(\Omega)}\left\|w_{\varepsilon, \lambda}\right\|_{L^{2}(\Omega)}+\left\|w_{\varepsilon, \lambda}-\lambda x\right\|_{H_{0}^{1}(\Omega)},
\end{aligned}
$$

so that $w_{\varepsilon, \lambda} v_{\varepsilon, i}^{\sigma} \longrightarrow w_{\lambda}^{*} u_{i}^{\sigma}$ strongly in $L^{2}(\Omega)$. Moreover, by (4.63) we have

$$
\begin{aligned}
\left\|\nabla\left(w_{\varepsilon, \lambda} v_{\varepsilon, i}^{\sigma}\right)\right\|_{L^{2}(\Omega)} & =\left\|w_{\varepsilon, \lambda} \nabla v_{\varepsilon, i}^{\sigma}+v_{\varepsilon, i}^{\sigma} \nabla w_{\varepsilon, \lambda}\right\|_{L^{2}(\Omega)} \\
& \leq\left\|w_{\varepsilon, \lambda}\right\|_{\infty}\left\|\nabla v_{\varepsilon, i}^{\sigma}\right\|_{L^{2}(\Omega)}+\left\|v_{\varepsilon, i}^{\sigma} \nabla w_{\varepsilon, \lambda}\right\|_{L^{2}(\Omega)} \leq c .
\end{aligned}
$$

By Lemma 3.2 and (4.61) we find that the first term of (4.59) converges,

$$
\left\langle-\Delta \psi_{\varepsilon}^{\sigma}, \varphi w_{\varepsilon, \lambda} v_{\varepsilon, i}^{\sigma}\right\rangle_{H^{-1}(\Omega), H_{0}^{1}(\Omega)} \rightarrow\left\langle\mu^{\sigma}, \varphi w_{\lambda}^{*} u_{i}^{\sigma}\right\rangle_{H^{-1}(\Omega), H_{0}^{1}(\Omega)}
$$

About the second term of (4.59), we observe that

$$
\begin{aligned}
\frac{1}{2} \int_{A_{\varepsilon}} \varphi \nabla P_{\varepsilon} \vartheta_{g_{i}}^{\varepsilon} w_{\varepsilon, \lambda} \nabla \psi_{\varepsilon}^{\sigma} & \leq\left\|\nabla P_{\varepsilon} \vartheta_{g_{i}}^{\varepsilon}\right\|_{\left(L^{2}\left(A_{\varepsilon}\right)\right)^{n}}\left\|\nabla \psi_{\varepsilon}^{\sigma}\right\|_{\left(L^{2}\left(A_{\varepsilon}\right)\right)^{n}}\left\|w_{\varepsilon, \lambda}\right\|_{L^{\infty}(\Omega)}\|\varphi\|_{L^{\infty}(\Omega)} \\
& \leq C\left\|\nabla P_{\varepsilon} \vartheta_{g_{i}}^{\varepsilon}\right\|_{L^{2}\left(A_{\varepsilon}\right)} .
\end{aligned}
$$

Moreover,

$$
\begin{aligned}
\left\|\nabla P_{\varepsilon} \vartheta_{g_{i}}^{\varepsilon}\right\|_{\left(L^{2}\left(A_{\varepsilon}\right)\right)^{n}}^{2} & =\int_{A_{\varepsilon}}\left|\nabla P_{\varepsilon} \widehat{\vartheta}_{g_{i}}\left(\frac{x}{\varepsilon}\right)\right|^{2} d x=2 \int_{A_{\varepsilon} \cap \Omega_{\varepsilon}}\left|\nabla \varepsilon \widehat{\vartheta}_{g_{i}}\left(\frac{x}{\varepsilon}\right)\right|^{2} d x \\
& =2 \frac{|\Omega|}{|\varepsilon Y|} \int_{A_{\varepsilon} \cap \varepsilon Y}\left|\nabla_{y} \widehat{\vartheta}_{g_{i}}\left(\frac{x}{\varepsilon}\right)\right|^{2} d x=2 \frac{|\Omega|}{|Y|} \int_{\frac{A_{\varepsilon}}{\varepsilon} \cap Y}\left|\nabla_{y} \widehat{\vartheta}_{g_{i}}(y)\right|^{2} d y,
\end{aligned}
$$

and as previously, by the absolute continuity of the integral, we have

$$
\left\|\nabla P_{\varepsilon} \vartheta_{g_{i}}^{\varepsilon}\right\|_{\left(L^{2}\left(A_{\varepsilon}\right)\right)^{n}}^{2} \longrightarrow 0, \quad \text { as } \varepsilon \longrightarrow 0 .
$$

So, as $\varepsilon \longrightarrow 0$, by (4.67), the integral

$$
\frac{1}{2} \int_{A_{\varepsilon}} \varphi \nabla P_{\varepsilon} \vartheta_{g_{i}}^{\varepsilon} w_{\varepsilon, \lambda} \nabla \psi_{\varepsilon}^{\sigma} \longrightarrow 0
$$

By (4.65) and (4.68) we obtain

$$
\frac{1}{2} \int_{\Omega} \varphi\left(\nabla P_{\varepsilon} u_{\varepsilon, i}^{\sigma}-\nabla P_{\varepsilon} \vartheta_{g_{i}}^{\varepsilon}\right) w_{\varepsilon, \lambda} \nabla \psi_{\varepsilon}^{\sigma} \rightarrow \frac{1}{2}\left\langle\mu^{\sigma}, \varphi w_{\lambda}^{*} u_{i}^{\sigma}\right\rangle_{H^{-1}(\Omega), H_{0}^{1}(\Omega)} .
$$


So, as $\varepsilon \longrightarrow 0$, by (4.48), (4.49), (4.50), (4.51), (4.52), and (4.69) the expression (4.46) tends to

$$
\begin{gathered}
\int_{\Omega}\left(\xi_{*, i}^{\sigma}-m_{Y^{c}}\left(\gamma_{i}\right)\right) \nabla \varphi w_{\lambda}^{*}+\frac{1}{2}\left\langle\mu^{\sigma}, \varphi w_{\lambda}^{*} u_{i}^{\sigma}\right\rangle_{H^{-1}(\Omega), H_{0}^{1}(\Omega)}-\int_{\Omega} \mathcal{A} \lambda \nabla \varphi u_{i}^{\sigma} \\
=\int_{\Omega} u_{i}^{\sigma} \varphi w_{\lambda}^{*}-\int_{\Omega}\left|u^{\sigma}\right|^{2} u_{i}^{\sigma} \varphi w_{\lambda}^{*}+\int_{\Omega} \theta f_{i} \varphi w_{\lambda}^{*} .
\end{gathered}
$$

Now if we take $\varphi \psi_{\varepsilon}^{\sigma}, \varphi \in \mathcal{D}(\Omega)$, as a test function in problem (4.43) we get

$$
\begin{aligned}
& \int_{\Omega}\left(\widetilde{\xi}_{\varepsilon, i}^{\sigma}-\widetilde{\gamma}_{\varepsilon, i}\right) \psi_{\varepsilon}^{\sigma} \nabla \varphi+\int_{\Omega}\left(\widetilde{\xi}_{\varepsilon, i}^{\sigma}-\widetilde{\gamma}_{\varepsilon, i}\right) \varphi \nabla \psi_{\varepsilon}^{\sigma} \\
& =\int_{\Omega} \widetilde{u}_{\varepsilon, i}^{\sigma} \varphi \psi_{\varepsilon}^{\sigma}-\int_{\Omega}\left|\widetilde{u}_{\varepsilon}^{\sigma}\right|^{2} \widetilde{u}_{\varepsilon, i}^{\sigma} \varphi \psi_{\varepsilon}^{\sigma}+\int_{\Omega} \chi \Omega_{\varepsilon}^{\sigma} f_{i} \varphi \psi_{\varepsilon}^{\sigma} .
\end{aligned}
$$

In a similar way to the previous case, we obtain for $\varphi \in \mathcal{D}(\Omega)$ as $\varepsilon \longrightarrow 0$

$$
\begin{gathered}
\int_{\Omega}\left(\xi_{*, i}^{\sigma}-m_{Y^{c}}\left(\gamma_{i}\right)\right) \nabla \varphi+\frac{1}{2}\left\langle\mu^{\sigma}, \varphi u_{i}^{\sigma}\right\rangle_{H^{-1}(\Omega), H_{0}^{1}(\Omega)} \\
=\int_{\Omega} u_{i}^{\sigma} \varphi-\int_{\Omega}\left|u^{\sigma}\right|^{2} u_{i}^{\sigma} \varphi+\int_{\Omega} \theta f_{i} \varphi
\end{gathered}
$$

and by a density argument it is true for $\varphi \in H^{1}(\Omega)$.

Taking $\varphi w_{\lambda}^{*}, \varphi \in \mathcal{D}(\Omega)$, as a test function in (4.72), we have

$$
\begin{aligned}
& \int_{\Omega}\left(\xi_{*, i}^{\sigma}-m_{Y^{c}}\left(\gamma_{i}\right)\right) w_{\lambda}^{*} \nabla \varphi+\int_{\Omega}\left(\xi_{*, i}^{\tau}-m_{Y^{c}}\left(\gamma_{i}\right)\right) \varphi \nabla w_{\lambda}^{*}+\frac{1}{2}\left\langle\mu^{\sigma}, \varphi u_{i}^{\tau} w_{\lambda}^{*}\right\rangle_{H^{-1}(\Omega), H_{0}^{1}(\Omega)} \\
& =\int_{\Omega} u_{i}^{\sigma} \varphi w_{\lambda}^{*}-\int_{\Omega}\left|u^{\sigma}\right|^{2} u_{i}^{\sigma} \varphi w_{\lambda}^{*}+\int_{\Omega} \theta f_{i} \varphi w_{\lambda}^{*} .
\end{aligned}
$$

From (4.70) and (4.73) we obtain

$$
\int_{\Omega}\left(\xi_{*, i}^{\sigma}-m_{Y^{c}}\left(\gamma_{i}\right)\right) \varphi \nabla w_{\lambda}^{*}+\int_{\Omega} \mathcal{A} \lambda \nabla \varphi u_{i}^{\sigma}=0
$$

Let us consider the vector valued function $\Psi=\left(0, \ldots, \varphi u_{i}^{\sigma}, \ldots, 0\right)$; by (4.33) and (4.34) we have

$$
\int_{\Omega} \operatorname{div}\langle\mathcal{A} \lambda, \Psi\rangle=\int_{\Omega}\left\langle\mathcal{A} \lambda, \nabla\left(\varphi u_{i}^{\sigma}\right)\right\rangle=0
$$

and as a consequence

$$
\int_{\Omega}\left\langle\mathcal{A} \lambda, u_{i}^{\sigma} \nabla \varphi\right\rangle=-\int_{\Omega}\left\langle\mathcal{A} \lambda, \varphi \nabla u_{i}^{\sigma}\right\rangle
$$

Then by (4.74) and (4.75) we obtain

$$
\int_{\Omega}\left(\xi_{*, i}^{\sigma}-m_{Y}\left(\gamma_{i}\right)\right) \varphi \lambda-\int_{\Omega} \mathcal{A} \lambda \varphi \nabla u_{i}^{\sigma}=\int_{\Omega}\left[\left(\xi_{*, i}-m_{Y}\left(\gamma_{i}\right)\right)-\mathcal{A} \nabla u_{i}^{\sigma}\right] \varphi \lambda=0,
$$


hence

$$
\xi_{*, i}^{\sigma}=\mathcal{A} \nabla u_{i}^{\sigma}+m_{Y^{c}}\left(\gamma_{i}\right) \quad \text { a.e. in } \Omega
$$

By the uniqueness of the solution of problem (2.6), convergences (4.13), (4.15), and (4.16) hold for the whole sequences $\left\{v_{\varepsilon}^{\sigma}\right\}_{\varepsilon}$ and $\left\{u_{\varepsilon}^{\sigma}\right\}_{\varepsilon}$, respectively. If now $\left\{\zeta_{\varepsilon}^{\sigma}\right\}_{\varepsilon}$ is any other family of uniform extensions of $\left\{u_{\varepsilon}^{\sigma}\right\}_{\varepsilon}$ bounded in $H_{0}^{1}(\Omega)$, we have $\zeta_{\varepsilon}^{\sigma}-u_{\varepsilon}^{\sigma}=0$ in $\Omega_{\varepsilon}^{\sigma}$ and it is bounded in $H_{0}^{1}(\Omega)$. Then, by Lemma 3.2, Theorem 2.1 is completely proved.

\section{Competing interests}

The authors declare that they have no competing interests.

\section{Authors' contributions}

The authors conceived and wrote this article in collaboration and with the same responsibility. All of them read and approved the final manuscript.

\section{Author details}

'Dipartimento di Ingegneria Elettrica e dell' Informazione, Università degli Studi di Cassino e del Lazio Meridionale, via G. Di Biasio 43, Cassino, 03043, Italia. ²Dipartimento di Scienze e Tecnologie, Universitá del Sannio, Via Dei Mulini 59/A Palazzo Inarcassa, Benevento, 82100, Italia.

\section{Authors' information}

The authors are members of the Gruppo Nazionale per l'Analisi Matematica, la Probabilità e le loro Applicazioni (GNAMPA) of the Istituto Nazionale di Alta Matematica (INdAM)

Received: 9 July 2014 Accepted: 19 September 2014 Published online: 07 October 2014

\section{References}

1. Tartar, L: Cours Peccot, Collège de France (March 1977). Partially written in F. Murat, H-Convergence, Séminaire d'analyse fonctionnelle et numérique de l'Université d'Alger (1977-78). English translation in Mathematical Modeling of Composite Materials, A. Cherkaev and R. V. Kohon (eds.), Progress in Nonlinear Differential Equations and their Applications, pp. 21-44. Birkhauser-Verlag (1997)

2. Cioranescu, D, Saint Jean Paulin, J: Homogenization in open sets with holes. J. Math. Anal. Appl. 71, 590-607 (1978)

3. Cioranescu, D, Murat, F: A strange term coming from nowhere. In: Cherkaev, A, Kohn, R (eds.) Topics in the Mathematical Modelling of Composite Materials, vol. 31, pp. 45-93. Birkhäuser, Basel (1997)

4. Allaire, G, Murat, F: Homogenization of the Neumann problem with non isolated holes. Asymptot. Anal. 7, 81-95 (1993)

5. Cardone, G, D'Apice, C, De Maio, U: Homogenization of mixed Neumann and Dirichlet problems. NoDEA Nonlinear Differ. Equ. Appl. 9, 325-346 (2002)

6. Cioranescu, D, Donato, P: Homogénéisation du problème de Neumann non homogéne dans des ouverts perforés. Asymptot. Anal. 1, 115-138 (1988)

7. Cioranescu, D, Murat, F: Un terme étrange venu d'ailleurs. In: Nonlinear Partial Differential Equations and Their Applications. Research Notes in Mathematics. Collège de France Seminar, vol. II, pp. 58-138, vol. III, pp. 157-178. Pitman, London (1981)

8. Cioranescu, D, Saint Jean Paulin, J: Homogenization of Reticulated Structures. Applied Mathematical Sciences, vol. 136. Springer, Berlin (2011)

9. Conca, C, Donato, P: Non homogeneous Neumann problems in domains with small holes. Modél. Math. Anal. Numér 22(4), 561-608 (1988)

10. Corbo Esposito, A, D'Apice, C, Gaudiello, A: Homogenization in a perforated domain with both Dirichlet and Neumann boundary conditions on the holes. Asymptot. Anal. 31(3-4), 297-316 (2002)

11. André, N, Shafrir, I: Minimization of the Ginzburg-Landau functional with weight. C. R. Math. Acad. Sci. Paris, Sér. I 321(8), 999-1004 (1995)

12. André, N, Shafrir, I: Asymptotic behaviour of minimizers for the Ginzburg-Landau functional with weight. Parts I and II. Arch. Ration. Mech. Anal. 142(1), 45-73, 75-98 (1998)

13. Bethuel, F, Brezis, H, Hélein, F: Asymptotic for the minimization of a Ginzburg-Landau functional. Calc. Var. Partial Differ. Equ. 1, 123-148 (1993)

14. Bethuel, F, Brezis, H, Hélein, F: Ginzburg-Landau Vortices. Birkhäuser, Basel (1994)

15. Beaulieu, A, Hadiji, R: Asymptotic for minimizers of a class of Ginzburg-Landau equation with weight. C. R. Math. Acad. Sci. Paris, Sér. | 320(2), 181-186 (1995)

16. Beaulieu, A, Hadiji, R: A Ginzburg-Landau problem having minima on the boundary. Proc. R. Soc. Edinb. A 128 123-148 (1998)

17. Beaulieu, A, Hadiji, R: Asymptotic behaviour of minimizers of a Ginzburg-Landau equation with weight near their zeroes. Asymptot. Anal. 22, 303-347 (2000)

18. Hadiji, R, Perugia, C: Minimization of a quasi-linear Ginzburg-Landau type energy. Nonlinear Anal., Theory Methods Appl. 71, 860-875 (2009)

19. Khruslov, E, Pankratov, L: Homogenization of boundary problems for Ginzburg-Landau equation in weakly connected domains. Adv. Sov. Math. 19, 233-268 (1994) 
20. Hadiji, R, Gaudiello, A, Picard, C: Homogenization of the Ginzburg-Landau equation in a domain with oscillating boundary. Commun. Appl. Anal. 7(2-3), 209-223 (2003)

21. Federer, $\mathrm{H}$, Ziemer, WP: The Lebesgue set of a function whose distribution derivatives are $p$-th power summable. Indiana Univ. Math. J. 22, 139-158 (1982)

22. Martio, O: John domains, bilipschitz balls and Poincaré inequality. Rev. Roum. Math. Pures Appl. 33, 107-112 (1988)

23. Attouch, H, Murat, F: Homogenization on fissured elastic materials, 85-03. Publications Avamac, Université de Perpignan (1985)

doi:10.1186/s13661-014-0223-2

Cite this article as: Faella and Perugia: Homogenization of a Ginzburg-Landau problem in a perforated domain with mixed boundary conditions. Boundary Value Problems 2014 2014:223.

Submit your manuscript to a SpringerOpen ${ }^{\circ}$ journal and benefit from:

- Convenient online submission

- Rigorous peer review

- Immediate publication on acceptance

- Open access: articles freely available online

- High visibility within the field

- Retaining the copyright to your article

Submit your next manuscript at $>$ springeropen.com 